\title{
SeeMore: a Kinetic Parallel Computer Sculpture for Educating Broad Audiences on Parallel Computation
}

\author{
Bo Li ${ }^{\mathrm{a}}$, John Mooring ${ }^{\mathrm{b}}$, Sam Blanchard ${ }^{\mathrm{c}}$, Aditya Johrid ${ }^{\mathrm{d}}$, Melinda Leko ${ }^{\mathrm{e}}$, \\ Kirk W. Cameron ${ }^{\mathrm{a}}$ \\ ${ }^{a}$ Dept. of Computer Science, Virginia Tech, Virginia \\ ${ }^{b}$ Microsoft, Seattle, Washington \\ ${ }^{c}$ School of Visual Arts, Virginia Tech, Virginia \\ ${ }^{d}$ Dept. of Information Sciences and Technology, George Mason University, Virginia \\ ${ }^{e}$ Dept. of Special Education, University of Kansas, Kansas
}

\begin{abstract}
We discuss the design, implementation, and evaluation of a 256-node Raspberry-Pi cluster with kinetic properties. Each compute node is attached to a servo mechanism such that movement results from local computation. The result is SeeMore, a kinetic parallel computer sculpture designed to enable visualization of parallel algorithms in an effort to educate broad audiences as to the beauty, complexity, and importance of parallel computation. The algorithms and interfaces were implemented by students from various related courses at Virginia Tech. We describe these designs in sufficient detail to enable others to build their own kinetic computing sculptures to augment their experiential learning programs. Our evaluations at exhibitions indicate $63 \%$ and $84 \%$ of visitors enjoyed interacting with SeeMore while $69 \%$ and $87 \%$ believed SeeMore has educational value.
\end{abstract}

Keywords: Parallel and distributed computing, kinetic art, computer science education

\section{Introduction}

Parallel and distributed systems are critical to our lives and livelihood. Services we rely upon daily such as search (e.g., Google, Bing), e-commerce (e.g., Amazon, Paypal), social media (e.g., Twitter, Facebook), entertainment (e.g., Netflix, Hulu), 5 and banking (e.g., CapitalOne, CitiBank) could not function without parallel and distributed processing on large scale systems in datacenters across the world. Scientific

Preprint submitted to Journal of Parallel and Distributed Computing

January 7, 2017

(C) 2017. This manuscript version is made available under the Elsevier user license http://www.elsevier.com/open-access/userlicense/1.0/ 
discoveries in health (e.g., gene manipulation) and medicine (e.g., drug discovery) leverage scientific computing that relies on parallel and distributed processing on supercomputers. Despite its importance, the average U.S. citizen knows very little about 10 parallel computation and how it affects them.

We describe a new approach to parallel and distributed computing (PDC) education for broad audiences. We have created a cluster computer in which compute nodes are attached to servo mechanisms that actuate in response to changes in workload. This enables dynamic, kinetic visualizations of the parallel computations that evolve in response to a parallel algorithm running on the system. In this way, viewers see and interact with the very computations that are purposely hidden deep within layers of software in services like Google search that they use everyday.

We identify three goals for our kinetic cluster: entertain and educate all audiences to improve the PDC pipeline; personalize the importance of parallel computation; and inspire participants to learn more. In particular, SeeMore attracts and compels current and future CS students to learn more about parallel computing and ultimately contributes to the pipeline for parallel and distributed computing education. We have incorporated the kinetic cluster in coursework at Virginia Tech for undergraduate, graduate, and local high-school students. These experiences range from asking students to program single system movement of a node to having students propose and implement new algorithms and interfaces for the full kinetic cluster. The SeeMore Vancouver and Manhattan algorithms and interfaces were implemented by undergraduate students from CS2506.

The 256-node SeeMore kinetic cluster is an experiential classroom of sorts. Just as students learn by visiting an art or science museum, SeeMore was designed to teach through an immersive experience. SeeMore has been visited by groups as varied as K-12 students, teachers, adults, seniors, girl scout troops, university presidents, legislators, and the director of the National Science Foundation. In these groups unfamiliar with parallel computing concepts our primary educational focus is imparting the basics of parallel computing and their importance. Our intent is to encourage and enable others to build prototypes of their own for themselves or their students to continue the experiential learning process SeeMore exemplifies. 


\section{Related Work}

SeeMore is a 256-node kinetic cluster where movement of Raspberry Pi computers attached to servo arms corresponds to parallel synchronous and asynchronous algorithms common to scientific and commercial applications. The SeeMore project was conceived to entertain and educate broad audiences on the importance of parallel computation to our everyday lives and to inspire exhibit participants to learn more. Although SeeMore is one of a kind, SeeMore combines computing visualization with

45 kinetic art for education. Thus related work falls into these two categories.

\subsection{Computing visualization for education}

Visualization tools have been used to educate audiences on complex topics in fields as diverse as Mathematics [22] and Nanoscience [29]. Others have used visualization to improve the teaching of various computer science topics. For example, Guo [13]

50 proposed programming visualization tools to teach Python. Egan and McDonald [9] developed similar tools for the C programming language. Karavirta and Shaffer [20] created an algorithm visualization library to ease web development. Fouh et al. [11] designed an interactive eTextbook to visualize computer algorithms. Velazquez-Iturbide et al. [34] focused on teaching greedy algorithms with visualization. Hundhausen et al. [16] analyzed the effectiveness of using visualizations for teaching algorithm courses. Mustafa [26] developed an interactive simulator to support teaching and learning of operating systems courses at the undergraduate level.

Hahn et al. [14] applied visualization techniques to interactive exploration of multithreaded software systems. Finlayson et al. [10] proposed a software framework for visualization of parallel programming concepts and execution. Offenwanger and Lucet [28] designed a visualization tool (ConEE) to teach the concepts of concurrent programming while aiding program debugging with interactive feedback. Visualization has also been used to aid computer scientists' analyses and optimizations of complicated topics [19, 21, 31, 27, 32, 8]. 


\subsection{Kinetic art for education}

Kinetic art [24] combines art and engineering often for education. Jee and Hong [18] investigated the effectiveness of a Science, Technology, Engineering, Art and Mathematics (STEAM) program for 5th and 6th grade children. Chung [5] studied the use of kinetic arts in robotics challenges for pre-college students in STEM subjects including computing.

Kinetic arts have been used to facilitate cross-disciplinary collaborations. Brunvand et al. [3, 2] showed students can benefit from collaboration between computer science and engineering and art students to create kinetic art. Lum et al. [23] used kinetic visualization to demonstrate that motion cues can improve the perception of $3 \mathrm{D}$ shapes and structures of statically engineered objects. Yilmaz et al. [37] showed kinetic art can benefit from collaboration with mechanics, mathematicians, etc.

\subsection{Our work in the context of prior work}

While visualization tools are effective for education of specific topics in computer science, we are not aware of any work that uses kinetic compute devices to explain basic parallel computing concepts to broad audiences. Most prior work uses different types of visualization (e.g. animation) or focuses on a very specific domain (e.g., robotics) or audience (e.g., specific course or grade). In contrast, our focus is on convincing broad audiences of the beauty, elegance, and importance of parallel computation in their everyday lives. Thus rather than teaching all aspects of a topic, we want to impart just enough information to excite and inspire an audience to continue their education on the topic of parallel computation. Therefore, the objectives of our SeeMore project are quite different from prior work.

While kinetic art has been used to promote education and collaboration with some success, to the best of our knowledge it has not been applied to parallel and distributed computing concepts. We believe art in service to education is an excellent means to broaden the appeal of complex topics to diverse audiences. In the SeeMore project, we combine the aesthetic aspects of a beautiful piece of art designed to be experienced with kinetic demonstrations that impart basic principals of parallel computation (paral- 
lelization, organization, elegance, and importance to every day life) to audiences of all backgrounds.

\section{Parallel Algorithms for Demonstration}

In order to captivate an audience, our kinetic sculpture must entertain as well as educate. Before we could design the physical system, we needed to complete two tasks related to the programming of the resulting kinetic cluster.

1. Identification of key algorithmic patterns used heavily by the commercial and scientific communities;

2. Selection of key algorithms with compelling visualizations that captivate an audience and tell a story that viewers can connect with.

To this end, we studied a number of parallel algorithms used in both scientific computing and large commercial data centers. We decided to focus on two types of straightforward parallel algorithms that fit the above criteria: synchronous and asynchronous. In this discussion, we assume a synchronous parallel algorithm follows a bulk synchronous parallel machine model [33] where tasks proceed in steps uninhibited between synchronization points (e.g. barriers). In contrast, we assume an asynchronous parallel algorithm follows a parallel random access model [17] where tasks proceed uninhibited by synchronization across tasks until completion.

\subsection{Synchronous Parallel Algorithms}

Simulation of the transport of subatomic particles is critical to many computational physics challenges. This problem has been widely investigated by the scientific community since transport sweeps are among the most time consuming steps in multiphase fluid flow analyses. Parallel transport sweeps can significantly improve the performance of multiphase fluid flow simulations by reducing the number of particles each subtask has to compute [25]. The Sweep3D application kernel [35, 15] benchmarks the performance of the neutron transport problem across various architectures [36, 12].

Given the wide acceptance and use of Sweep3D, we adopted the compelling patterns of sweep algorithms for visualization in SeeMore. To diversify the patterns and 
represent various types of network- or system-specific implementations, we studied and implemented row-wise sweeps, column wise sweeps, diagonal sweeps (without pipelining), wave-front sweeps, and row/column-wise odd-even sweeps. Figure 1 illustrates the patterns that result from the kinetic movement on a 2 dimensional grid where squares represent Raspberry Pi's attached to controllable servos. For example, synchronous row-wise sweep (see Figure 1a) propagates its row-synchronized communication from the top row of Raspberry Pi's to each succeeding (lower) row until it reaches the bottommost row. In this deterministic pattern, communication propagates only when all Raspberry Pi's in a row complete their compute step.

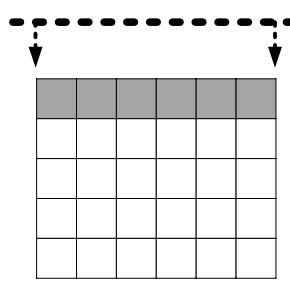

(a)

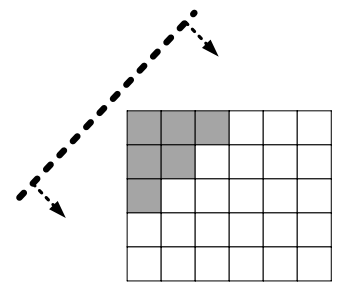

(d)

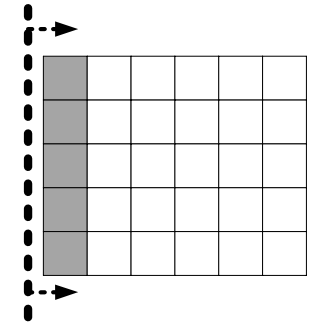

(b)

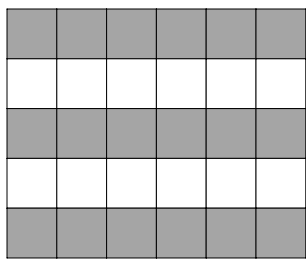

(e)

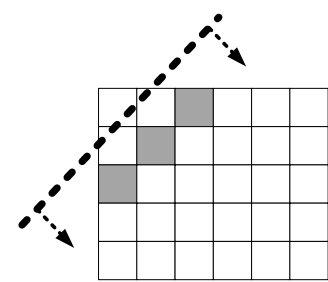

(c)

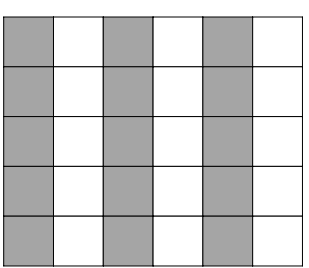

(f)

Figure 1: Sweep patterns in SeeMore. (a): row-wise sweep; (b): column-wise sweep; (c): diagonal wavefront sweep; (d): diagonal regular sweep; (e): row-wise odd-even sweep; (f): column-wise odd-even sweep.

\subsection{Asynchronous Parallel Algorithms}

Though scientists may be familiar with synchronous algorithms, the general public may not. Twitter, Google, Facebook and other big-data and cloud computing endeavors often rely on asynchronous algorithms to provide their services. In these discussions, 
dent tasks such as the threads of a parallel search can be distributed somewhat arbitrarily to multiple servers. Each server works independently to complete the assigned tasks and returns results as they become available asynchronously in relation to other tasks.

to modern data centers. In its most basic sense, as described to SeeMore audiences, MapReduce constitutes just two key phases: map and reduce. In the map phase, multiple servers are used to filter and sort the data under study. In the reduce phase, a reduction or summary of the information from all the distributed systems is produced.

\section{Design of SeeMore}

SeeMore was designed to be both beautiful and functional. We began with the design of a basic kinetic unit, or movable Raspberry Pi computer, and then used these units to create a programmable kinetic cluster that demonstrates the patterns described in the previous section. We developed three SeeMore prototypes composed of 1, 9, and the design which led to iterative improvements resulting in the final design of the 256node SeeMore kinetic sculpture. 


\subsection{The basic kinetic unit}

The visualization of the kinetic sculpture is highly dependent on the design of each kinetic unit. Figure 2 shows the design of a single kinetic unit. The movement of each node in the cluster is locally controlled and articulates (or moves outward) in proportion to its individual processing load. We map the runtime workload of each computing node to a discrete degree of rotation of the servo mechanism that controls the resulting angle of the Raspberry Pi kinetic unit. Each kinetic unit is a fully functional compute node including a compute/control unit with memory capable of running programs; a servo motor that drives the rotation or extension of the kinetic unit; and a control mechanism (i.e. control arm, gears, etc.) enabling controlled motion of the kinetic unit.

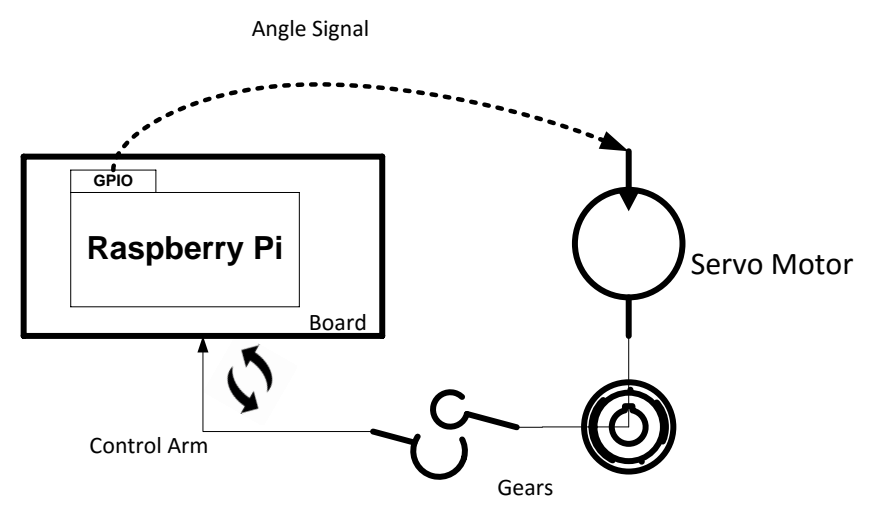

Figure 2: A basic, 1-node, kinetic unit for SeeMore.

We use a Raspberry Pi computer model B/B+ running Moebius Linux as the control unit. Raspberry Pi was selected for its small form factor, light weight, low price, and high availability. We use available Raspberry Pi system tools including GNU compilers, Java virtual machines, Python, Bash, Qt, etc.

Each Raspberry Pi is mounted to an arm plate and movable arm which is in turn attached to a servo motor. We selected a HITEC HS-5485HB servo motor that provided necessary functionality at a decent price point. We used the 40 Raspberry Pi general purpose input/output (GPIO) pins and available libraries to control the corresponding 
servo motor for each kinetic unit. The servo motor drives the control arm through mechanical linkages to turn the faceplate at the end of the control arm to about a 45 degree angle relative to its resting position flat against the cluster frame. The Raspberry $\mathrm{Pi}$ is mounted to the faceplate. The servo is capable of moving about 90 degrees in our design.

We calibrate the maximum angle for the servo and map Raspberry Pi workload utilization to a discrete set of angles between zero and the maximum. For example, when a Raspberry Pi encounters a moderate workload, CPU utilization maps to a discrete rotation setting for the servo motor which moves the associated Raspberry Pi, faceplate, and arm to a proportional angle. When the system returns to idle, the Raspberry Pi, faceplate and arm return to the resting position against the cluster frame.

\subsection{Prototype I}

Once we completed and tested the functionality of a 1-node, basic kinetic unit, we set out to create a 9-node version to study the coordination of kinetic movement across nodes. We assembled 9 basic kinetic units into a $3 \times 3$ 2-dimensional aluminum extrusion grid designed by the artistic team (see Figure 3 .

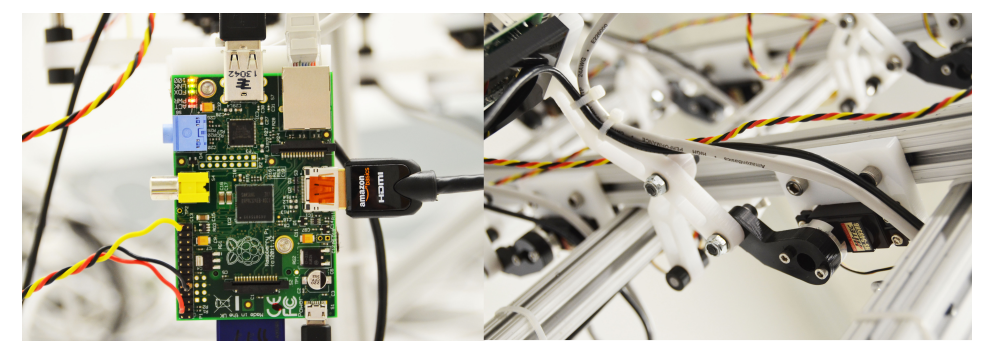

Figure 3: Prototype I.

All the Pi's were connected to a central desktop server through a 1 Gigabyte Ethernet switch. The server desktop provides a gateway for remote access to the 9-node local network of Raspberry Pi's. Parallel applications can be launched from either the server or any of the Raspberry Pi systems. Other than the choice of hardware, the system is set up as a traditional cluster and parallel applications can be launched from either one of the Pi's, or the desktop server. 
This prototype enabled debugging of the base environment for parallel program-

ming of the kinetic system. The system was configured to execute pthreads, OpenMP, MPI, and sockets via Ethernet.

After the system was fully functional, we began implementing the aforementioned synchronous algorithms to test the hardware capabilities, timing, coordination, etc. We identified a number of issues to be addressed in the next 30-node prototype:

- Initially, the resulting motion of a Raspberry Pi in response to its workload was shaky and susceptible to damage. This required a redesign of the arm from a single plastic arm (see Figure 3 ) to a double plastic arm for additional stability.

- Initially, the servos reacted too quickly to changes in workload. This required adjusting the refresh rate for servo changes. A similar problem manifested itself in programs where workload intensity varied so frequently that the servo could not respond without quickly becoming overworked and malfunctioning. This was handled by dampening the response rate of the servo mechanism more in line with the typical response rate of a mechanical device. For this reason, algorithms displayed on the kinetic sculpture are actually running much slower (at a rate acceptable to the servo) than the compute rate of the Raspberry Pi computer.

- After longer runs, we found the Raspberry Pi's were susceptible to a number of forms of corruption rendering them non-functional. In one case, the SD cards were corrupted and at times had to be rebooted, re-imaged, or replaced. This was likely due to the purchase of cheaper SD cards and with a refresh of higher quality cards, these occurrences happened much less frequently. Nonetheless, we always keep a card burner nearby when the large system is running at an exhibit. We also took steps to streamline diagnosis and handling of SD failures.

- During longer runs we would at times lose systems randomly for no obvious reason. After much testing we deduced that power fluctuations were the likely culprit. We installed power conditioners on the system which reduced the occurrences drastically. However, we later learned that the same problem manifested from lower quality USB power cables. Purchasing higher quality cables reduced 
these faults yet again.

- During longer runs we also noticed that some of the servos would break completely. We found that the weight and tension of the USB and Ethernet cables resulted in high amounts of torque on the servos causing them to at times overheat and wear out faster. This resulted in a redesign of the cabling to reduce the torque. For example, we purchased right-angle connectors for the USB cables to remove bends at the connection point on the Raspberry Pi's.

\subsection{Prototype II - Lil' SeeMore}

The 30-node prototype, later named "Lil' SeeMore" or "lil' SeeMore", was originally designed as another disposable prototype. We had learned so much from the 9-node prototype that there was concern we were not ready to scale to 256 nodes. We anticipated there were more lessons to learn with a moderate amount of scale at 30-nodes. In the end, the 30-node structure achieved full functionality though we still made adjustments. The result was a 30-node, more portable version of the full SeeMore cluster. Lil' SeeMore has made several exhibit appearances and was the subject of a previous study [4] of a select group of 8th graders.

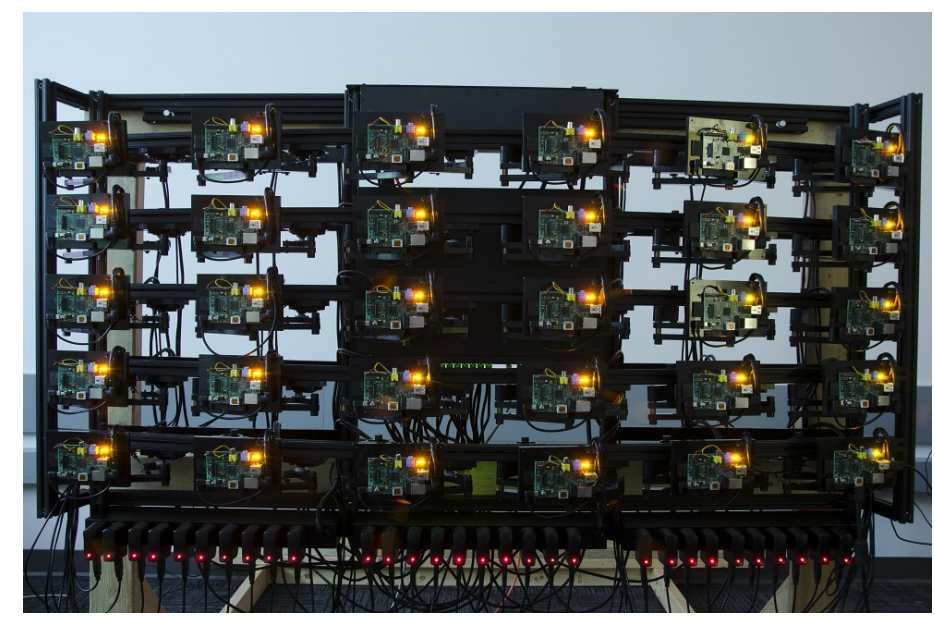

Figure 4: Prototype II: lil’ SeeMore

Figure 4/shows the lil' SeeMore kinetic sculpture. Lil' SeeMore has many aesthetic 
improvements. This design leverages an updated single kinetic unit with a scissor shaped, doubled control arm for extra stability during movement. The sculpture has all black components from the aluminum extrusion frame to the scissor arms and wiring. Even the ethernet switch, power conditioner and central server (a black Mac Mini 2014) were selected for functionality, form factor and color (i.e. black).

All parts were custom designed from the more than 30 plastic and metal pieces necessary to assemble a single arm for a kinetic unit to the cuts and curvature of the aluminum extrusion framing and the custom USB and Ethernet wiring. To reflect light, our artistic team added semi translucent acrylic covers on top of each Raspberry Pi. The number of Raspberry Pi's (30 total) was chosen for both aesthetics (a $5 \times 6$ grid) and for testing (about 3x more nodes versus the previous prototype).

This design was not issue free. In some instances, we suffered from problems similar to those encountered in the 9-node design. The biggest issue for lil' SeeMore was the discovery that an upgrade from the Raspberry Pi Model B (in the initial version of lil' SeeMore) to the Model B+ (in a later version of SeeMore) precluded the use of the GPIO pins of the Raspberry Pi to power the servos. This effectively resulted in a doubling of the number of cables in the cluster. While this wasn't particularly onerous for 30-nodes, it would become an issue at 256-nodes due to added cost, design time, and assembly time.

\subsection{Prototype III - SeeMore}

270 Lil' SeeMore was a prototype to test implementation theories and tweak our designs before moving to a larger scale. The 256-node SeeMore kinetic sculpture (see Figure 57 was designed to be experienced. The scale has a purpose. Data centers and supercomputers are tremendous in size, so we believed it necessary to impress upon exhibit attendees that these are huge machines that can seem daunting but perform noble services we rely upon. Moreover, the larger scale leads to more dramatic algorithmic patterns that are at once more obvious and more interesting.

Our artistic team also wanted the user to be lured from afar by the scale but then to have a different experience when up close. The large design has a cylindrical, hourglass shape that quite literally leans over people as they approach. When very close, the 
sound of the servos and the motion of each Raspberry Pi is almost mesmerizing creating a very different experience.

SeeMore is a 256-node version of lil' SeeMore with minor changes. Due to availability issues we had to migrate to the Raspberry Pi Model B+ just two weeks before the final assembly of SeeMore. The Model B was used in lil' SeeMore as mentioned.

285 This caused havoc as the previous servo drivers from the Model B did not work on the Model B+. Additionally, this is where the power issue with GPIO pins manifested itself dramatically.

We used another Mac Mini as the central server for SeeMore. The migration to the Model $\mathrm{B}+$ also resulted in the switch to micro-SD cards requiring re-imaging of all 25616 GB SD cards with the OS and tool chain. We use 648 -port Ethernet switches for the network. SeeMore also requires 4 15-Amp power conditioners to protect all the Raspberry Pi's and servo motors from power surges. The sculpture uses 26 power strips for power delivery. The power strips have 256 dual-slot 5 Volt power supplies plugged into them.

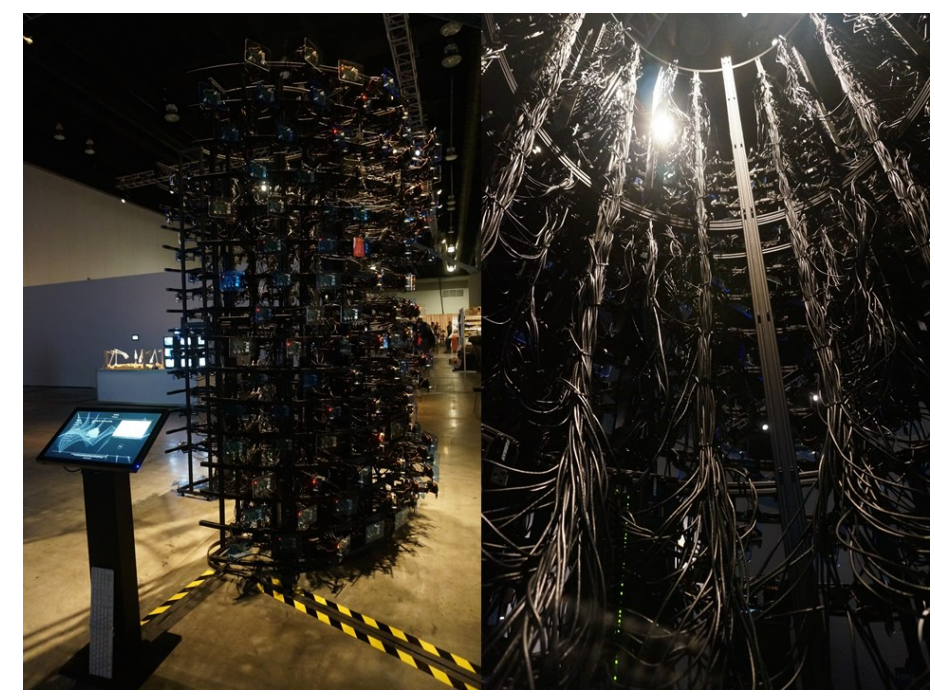

Figure 5: Prototype III: SeeMore

295 SeeMore also requires: 
- 2200 feet of customized Ethernet cable;

- 1320 feet of aluminum extrusion;

- 1563 feet of USB cable;

- 1152 inches of threaded rod;

- 7312 pieces of hardware (e.g. screws, nuts, and washers);

- 580 pounds of CNC machined plastic mechanical components;

- 2048 bronze bearings.

\section{Software Interfaces}

\subsection{Lil' SeeMore Interface Overview}

305 parallel algorithms of any pattern including the synchronous and asynchronous algorithms discussed. Lil' SeeMore was designed to show others how these algorithms work. Thus, the original interface (see Figure 6) was an ambitious, interactive design much like a series of tutorials designed for direct interaction and control by users. rotates through synchronous algorithm patterns.

The introduction video demonstrates several synchronous algorithms discussed in Section 5.2 Afterward users can view a MapReduce tutorial (discussed in Section 5.3 that has screen depictions of the MapReduce algorithm in movement. After the tutorial, the audience can take a short quiz to test their knowledge of basic concepts of parallel computing. 


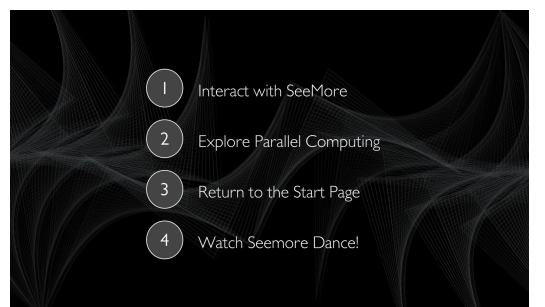

(a) Main menu page.

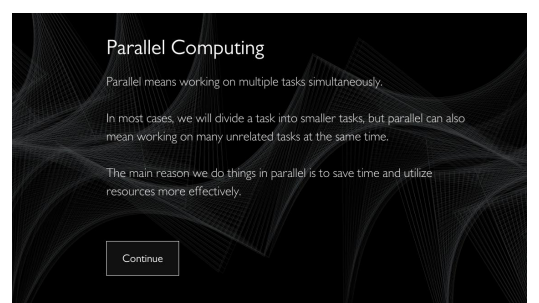

(b) Tutorial page.

Figure 6: Tutorial GUI of lil' SeeMore.

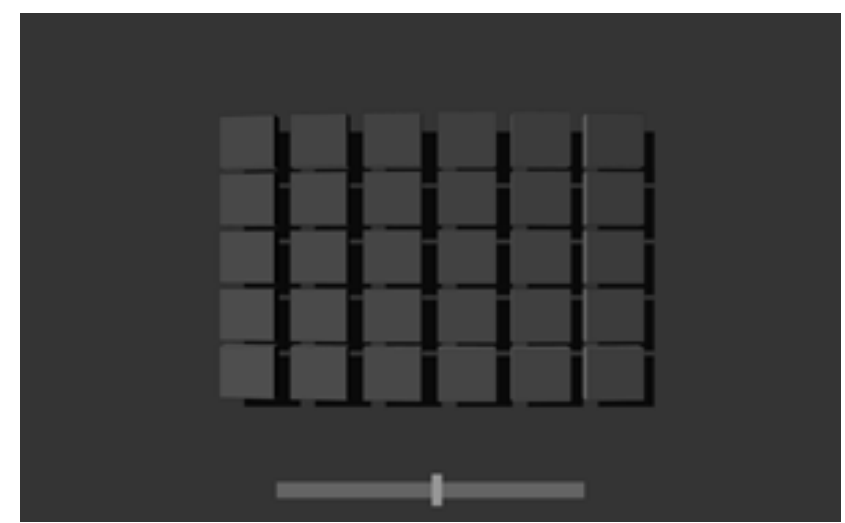

Figure 7: Interactive GUI of lil' SeeMore.

\subsection{Synchronous Algorithms on lil' SeeMore}

As discussed in Section 3 , we selected a number of sweep algorithms for visualization on the kinetic sculptures. In our demonstration, the movement of the kinetic units corresponds to six patterns: row-wise sweeps, column wise sweeps, diagonal sweeps (without pipelining), wave-front sweeps, and row/column-wise odd-even sweeps.

We implemented the sweep algorithms using $\mathrm{C}++$ and the Message Passing Interface (MPI). Once a Raspberry Pi starts performing its work, the kinetic unit moves to a precise angle. When the assigned work is complete, each kinetic unit moves back into a resting position flush against the sculpture.

Figure 8 a shows a cartoon of a column-wise sweep followed by the correlated phys- 
ical movement of the first column on lil' SeeMore (Figure $8 b$ ) followed by the second column on lil' SeeMore (Figure 8c). Figure 9 shows a similar series of the 3rd and 4th diagonals of a diagonal wavefront sweep on lil' SeeMore. As the kinetic units move on the physical sculpture, a synchronized animation appears on the touch screen where each block corresponds to a kinetic unit (not shown). The live demonstration video of all the sweep patterns is available on YouTube ${ }^{1}$. This lil' SeeMore demonstration never appeared formally in the extent literature but was submitted to compete for (and ultimately won) an exhibit space for SeeMore at the SIGGRAPH 2014 Art Gallery Exhibition ${ }^{2}$ in Vancouver, Canada [1].

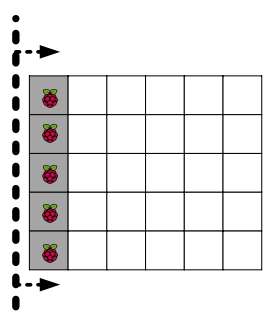

(a) Sweep pattern.

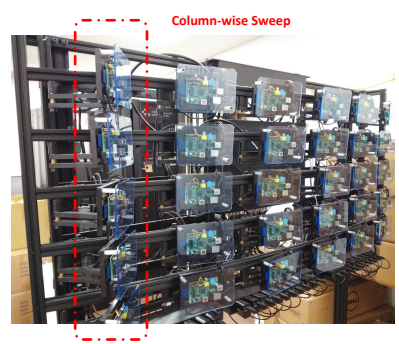

(b) The first column.

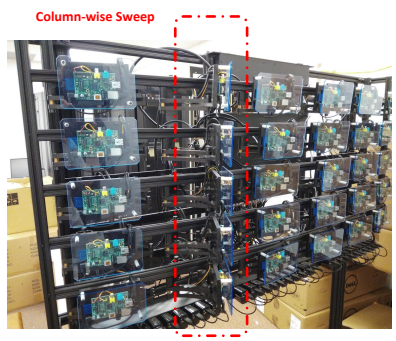

(c) The second column.

Figure 8: Visualization of column-wise sweep.

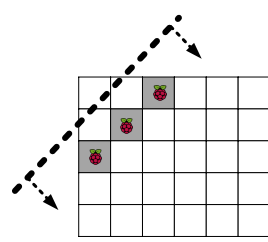

(a) Diagonal wavefront pattern.

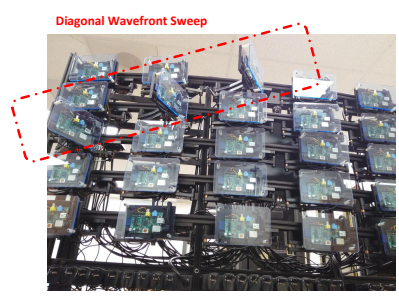

(b) 3rd diagonal line.

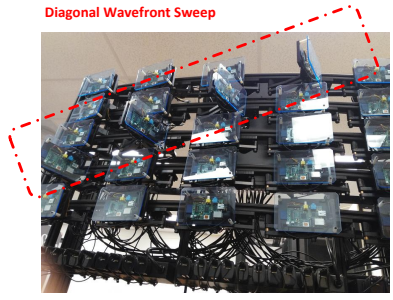

(c) 4th diagonal line.

Figure 9: Visualization of diagonal wavefront sweep.

\footnotetext{
${ }^{1}$ https://www.youtube.com/watch?v=5fp4-PBNHpk

${ }^{2} \mathrm{http} / / /$ s2014.siggraph.org/attendees/art-gallery/events/SeeMore
} 


\subsection{Asynchronous Algorithms on lil' SeeMore}

Lil' SeeMore was designed to directly interact with visitors. Thus, we designed a MapReduce tutorial that describes each stage of the algorithm and moves Raspberry Pi's corresponding to each stage of the algorithm. This demonstrates how the collection of nodes cooperate to provide the service.

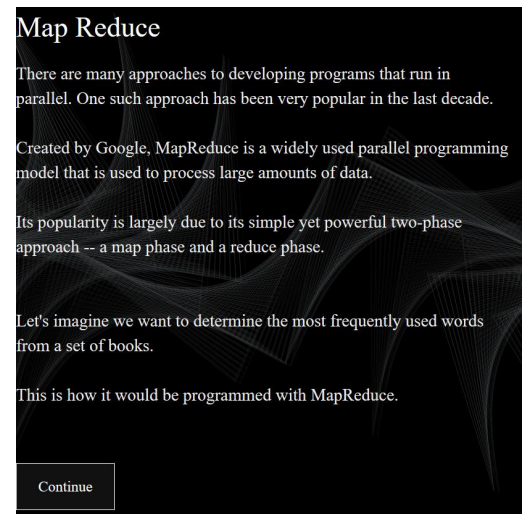

(a)

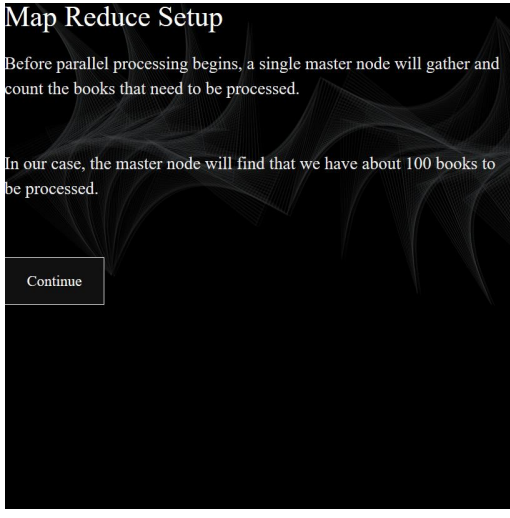

(b)

Figure 10: Keyword sorting using MapReduce.

We implemented MapReduce to conduct a parallel keyword sort. Beginning with the text from 100 books, the code determines the most frequently used words. Figure 10 shows screen captures of the interfaces that introduce the algorithm, identify the master node via movement on the cluster, and describe the initial setup.

As mentioned, MapReduce has two primary phases: map and reduce. Figure 11 shows the map phase interface and the corresponding visualization on lil' SeeMore. We use 18 Raspberry Pi's as the mapper nodes or the bottom 3 rows of lil' SeeMore. Figure $11 \mathrm{~b}$ demonstrates how mapper nodes extend when counting word frequency for a subset of the text from the 100 books.

Once all nodes complete their counting, the map phase has completed. In the demo this event awaits user input to "continue" the tutorial. In non-interactive mode, this synchronization can be eliminated and nodes can proceed immediately to the reduce phase. In this demo, once the computations are complete and the user touches "continue", the system proceeds to the reduce phase. 
The reduce phase ensures that the results of all the mapper nodes are aggregated. In this demonstration, we use the remaining 12 Raspberry Pi's or the top 2 rows of lil' SeeMore to perform the reduction. Figure $11 \mathrm{~b}$ demonstrates how these nodes extend when processing the reduction of the data. Upon completion of this phase, and in response to the user touching "continue", the results of the word count appear on the screen.

The tutorial ends with a final set of screens that implement the same counting algorithm on a single Raspberry Pi node. This results in a single Raspberry Pi moving and remaining out and therefore active for a period of time that exceeds the time for the parallel algorithm. This corresponds with an explanation on screen discussing the need for parallel computing for large data sets.

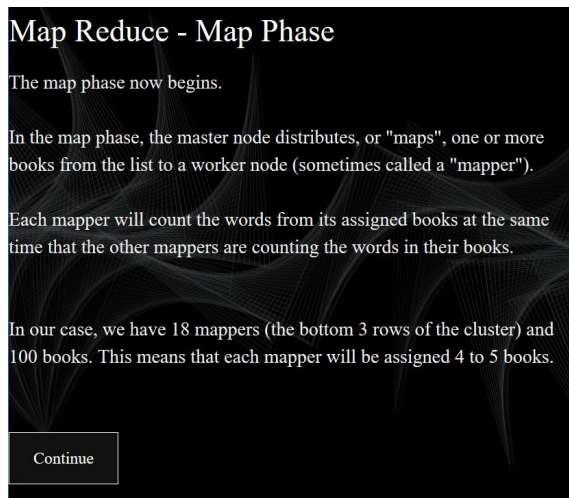

(a) Tutorial interface.

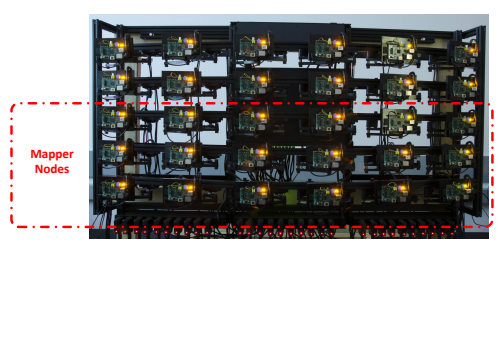

(b) Kinetic visualization.

Figure 11: Map phase of MapReduce on lil' SeeMore.

\subsection{Asynchronous Algorithms on SeeMore}

SeeMore, the 256-node kinetic sculpture, was created for large audiences. SeeMore was designed to carry out both synchronous and asynchronous algorithms. Aside from the scale, the synchronous sweep algorithms on SeeMore perform in much the same way as those on lil' SeeMore. We felt the compelling visuals of these algorithms spoke for themselves and did not require a corresponding on-screen visual aid.

The asynchronous MapReduce algorithm, without interactive pauses as described 


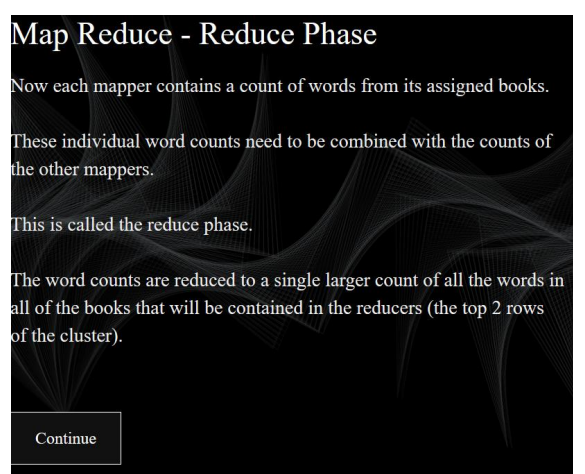

(a) Tutorial interface.

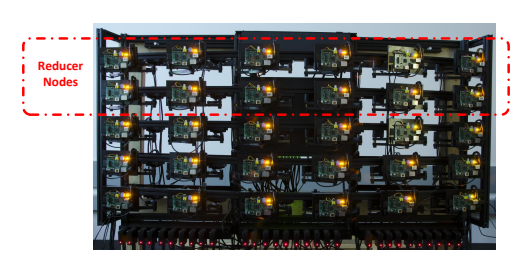

(b) Kinetic visualization.

Figure 12: Reduce phase of MapReduce on lil' SeeMore.

in the lil' SeeMore demo, is harder to follow and understand without a corresponding on-screen demonstration. Furthermore, connecting SeeMore to a use case similar to a Google search was deemed critical to tell a story that convinces an audience as to the importance of parallel computation to their every day lives. Additionally, we designed an interface that could be customized for a locale to make an even deeper connection with exhibit attendees.

\subsubsection{The Vancouver Algorithm}

We implemented a home-grown version of parallel search for SeeMore similar to MapReduce. For our first exhibition at SIGGRAPH 2014 in Vancouver, Canada, we identified an online database managed as part of the City of Vancouver Open Data Catalogue ${ }^{3}$ that specifies tree types by location for the greater Vancouver area.

The head node, our Mac Mini, initiates the search and distributes the keyword (a neighborhood name) to the 256 Raspberry Pi's. The neighborhood is highlighted on screen (see Figure 13) in a Vancouver map as the search begins. As the search proceeds the 256 nodes move to symbolize the activity on each node. Computation makes the kinetic nodes move outward when they are busy with their assigned tasks. The compute cluster counts the number of trees of each type in the given neighborhood then goes

\footnotetext{
${ }^{3}$ http://data.vancouver.ca/datacatalogue/streetTrees.htm
} 
idle. Next, each node conducts the reduce step to symbolize aggregation of the results on the head node and the tree counts for the given neighborhood appear on screen. The demonstration is designed to loop through dozens of neighborhoods and repeat for the duration of the exhibit.

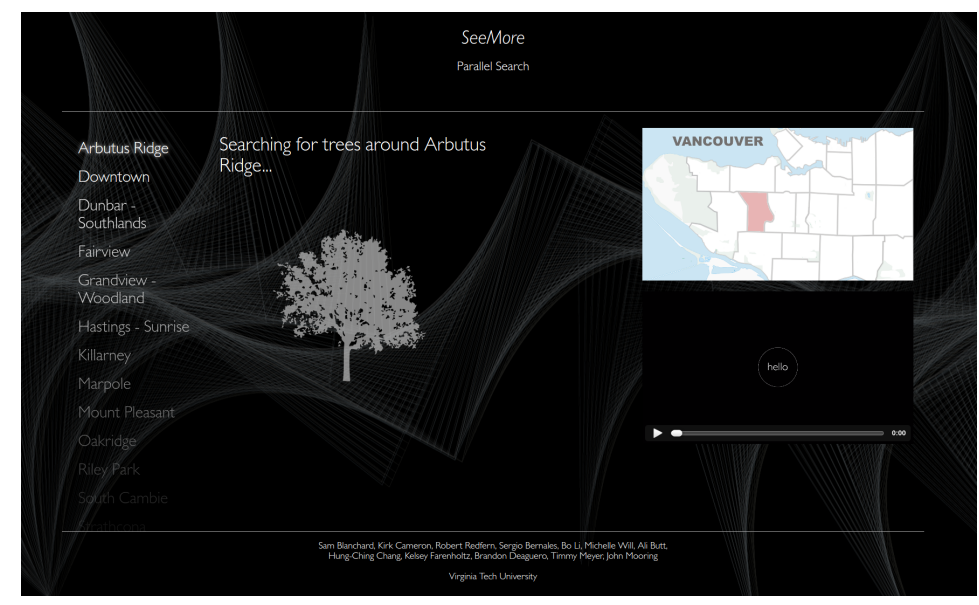

Figure 13: Vancouver Tree Searching GUI.

\subsubsection{The Manhattan Algorithm}

SeeMore's second large installation was at the 2015 International Maker Faire in New York City, New York. We made several enhancements to the software interface based upon lessons learned at SIGGRAPH 2014. For the synchronous algorithms, we selected one at random for a performance in between each live demo of parallel MapReduce. This enabled us to pipeline large groups from the live demo to close up interaction with the sculpture. For the MapReduce demonstration, we wanted an interface that resonated with the New York audience and was obviously connected to what was happening on the kinetic sculpture during the parallel computation.

For this exhibit of SeeMore, we identified a geospatial database maintained by the New York City Open Data project 4 . This database contains geospatial coordinates for points of interest in the 5 boroughs of New York City. Coordinates are given for

\footnotetext{
${ }^{4}$ https://nycopendata.socrata.com
} 
trees, wifi hotspots, bike shelters, pay phones, theaters, breweries, rat sightings, and restaurant health inspection grades. We designed an algorithm similar to the Vancouver Tree Algorithm.

In this instance, the demonstration starts with a subway stop name within the 5 boroughs and the parallel MapReduce-based algorithm identifies and counts points of interest within a mile radius of this stop. Figure 14 shows a screenshot of the display providing the name of the subway stop to be searched and highlights the borough it lies within.

After a brief pause, the algorithm begins execution on the system and the on screen image changes to a dynamic 3D rendering of SeeMore (see Figure 15. Initially, the image is of a cylindrical shape like the sculpture but it quickly unfolds to resemble a large 2D grid of 256 blocks not unlike the grid in the lil' SeeMore rendering. The blocks in the grid change color to reflect what is happening during the Idle phase (grey), Map phase (green), and Reduce phase (blue) of the algorithm. When each node completes all phases, the entire figure returns to Idle (grey) and the screen again reverts to the borough map along with the results from the parallel search. After a short delay allowing a random synchronous sweep pattern to execute across the kinetic sculpture, a new subway stop is identified and the demonstration repeats.

The front end graphical interface was implemented using a mixture of HTML and the Processing Graphics API. The initially limited performance of 3D graphics in WebGL necessitated that the UI be ported over to a native framework for which Qt was selected. HTML was rendered through Qt's port of WebKit, and the 3d portion of the UI was rendered using CProcessing and then embedded in HTML by way of Qt's Webkit Widgets. This allowed the same code to run with greatly enhanced performance. The UI code runs on top of a backend written in $\mathrm{C}++$ and Python that takes care of executing jobs on the sculpture and handling bidirectional UDP communication between the nodes and the front end for real time visualizations and user interaction with the sculpture. 


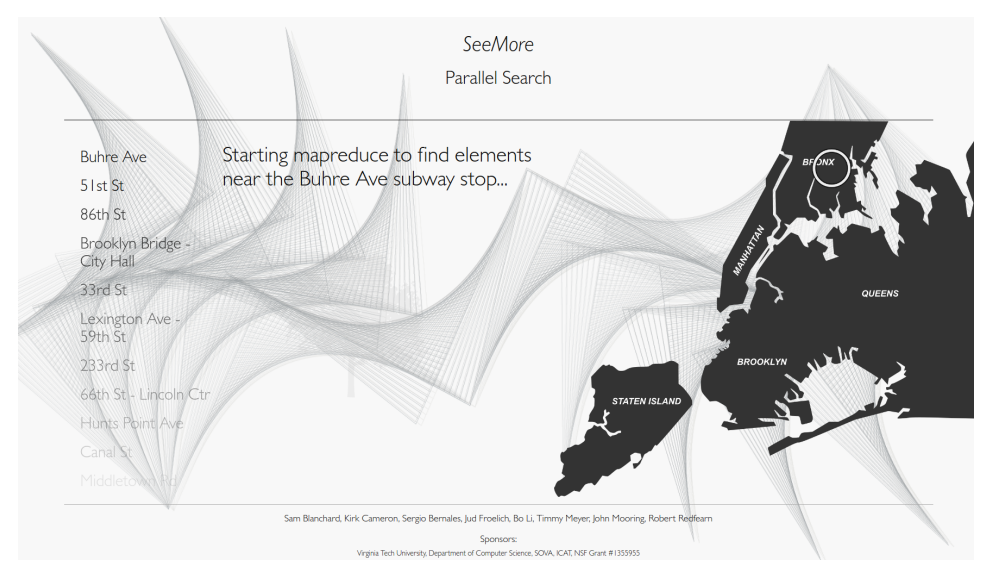

Figure 14: NY Manhattan Database GUI.

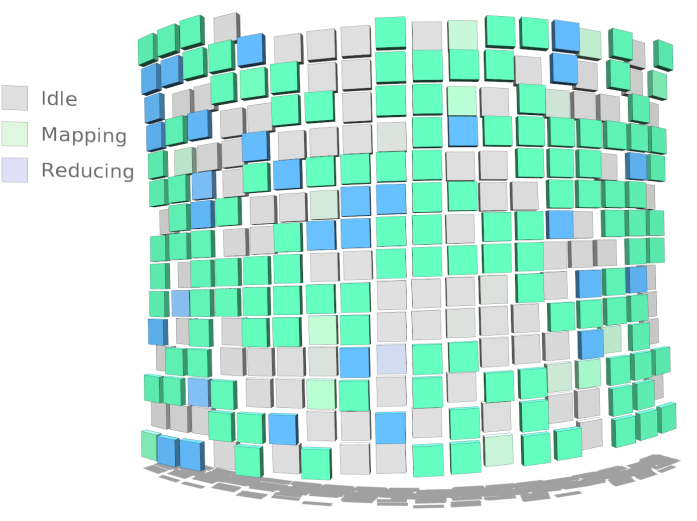

Figure 15: Search Job Visualization.

\section{Assessment}

The objective of the SeeMore project is to create interactive exhibits and demonstrations that:

- O1. Entertain and educate all audiences to improve PDC pipeline;

- O2. Personalize the importance of parallel computation; and

- O3. Inspire participants to learn more.

445

We employ both qualitative methods [6, 30] and quantitative methods (e.g., surveys designed by education researchers) to evaluate the ability of the SeeMore interactive 
exhibit to meet the aforementioned objectives [O1, O2, O3].

\subsection{Stage I Assessment: Early Lessons from Lil’ SeeMore (30-node cluster)}

We primarily used qualitative assessments and interviews with participants to as-

sess the impact of the smaller 30-node SeeMore cluster (a.k.a. lil' SeeMore) on audiences. Lil' SeeMore was exhibited in the Moss Center at Virginia Tech over a two-week period (May 3-18, 2014) with between 300 and 500 visitors in total. The details of our qualitative assessment of the ability of the kinetic sculpture to inform a small group of local 8th grade students on basic parallel computing concepts can be found in our related paper [4].

These early studies were limited in scope and evaluation of parallel computation learning objectives focused on "divide and conquer" algorithms and parallel computation patterns on the smaller 30-node kinetic sculpture. No scientific studies of broader audiences were conducted. Our experience with these exhibits and audiences led to a number of observations that influenced the design of the study and evaluation of the larger 256-node kinetic cluster. Overall, we found our assessment methods would need extensions to effectively evaluate our project objectives at scale.

Specifically, after several small exhibits with at most 50 people at a time, we determined that the interactive nature of the 30-node tutorials and touch screen would not scale well beyond 1-4 participants at a time. Experiencing all the tutorials and interactive touch screens took from 5 to 10 minutes. For the 256-node kinetic sculpture exhibit, we were targeting large exhibitions from 15,000 to 350,000 attendees. We estimated the need to educate between 300 and 600 visitors per hour (i.e., crowds two orders of magnitude larger than early exhibits of the 30-node system). We concluded that the large 256-node kinetic cluster would require improved, less-interactive interfaces and tutorials to ensure the exhibit could meet our objectives and handle audiences as large as $30-50$ people every $2-4$ minutes.

\subsection{Stage II Assessment: Evaluations of SeeMore at Scale}

The 256-node SeeMore cluster (a.k.a. SeeMore) is designed to meet the aforementioned objectives [O1, O2, O3] for large audiences. To date we have presented 
Table 1: IMF 2015 and SEF 2016 Survey Questions. Survey questions provided to exhibit attendees at the annual International Maker Faire (IMF), New York Hall of Science, New York City, New York, September 21-27, 2015 ( 90,000 attendees) and the biennial U.S.A. Science and Engineering Festival (SEF), Washington, D.C., April 15-19, 2016 (365,000 attendees). Participants were asked to fill out surveys after a short 1-2 minutes demonstration and $\mathrm{Q} \& \mathrm{~A}$ with the SeeMore design team. $[O 1=$ Entertain and educate all audiences, $\mathrm{O} 2=$ Personalize the importance of parallel computation, $\mathrm{O} 3=$ Inspire participants to learn more.]

\begin{tabular}{|c|c|c|c|c|}
\hline & \multicolumn{3}{|c|}{ SeeMore Project Objectives } \\
\hline & & O1 & $\mathrm{O} 2$ & $\mathrm{O} 3$ \\
\hline \multirow{13}{*}{ 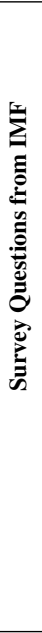 } & $\begin{array}{l}\text { 1. Prior to interacting with SeeMore how familiar were you with the } \\
\text { concept of parallel computing: }\end{array}$ & & $\checkmark$ & \\
\hline & $\begin{array}{l}\text { 2. Now that you have interacted with SeeMore, how would you rate } \\
\text { your level of understanding of parallel computing: }\end{array}$ & $\checkmark$ & $\boldsymbol{V}$ & \\
\hline & $\begin{array}{l}\text { 3. Indicate whether you (1) strongly agree, (2) agree, (3) disagree, or } \\
\text { (4) strongly disagree with the following statements (Circle/Cross One): }\end{array}$ & & & \\
\hline & a. I now better understand how things work in parallel: (1) (2) (3) (4) & $\boldsymbol{V}$ & & \\
\hline & $\begin{array}{l}\text { b. The computational patterns displayed by SeeMore helped my visual } \\
\text { understanding of parallel computing: (1) (2) (3) (4) }\end{array}$ & $\boldsymbol{v}$ & & \\
\hline & c. I enjoyed interacting with SeeMore: (1) (2) (3) (4) & $\boldsymbol{V}$ & $\boldsymbol{V}$ & $\boldsymbol{V}$ \\
\hline & d. I would like to learn more about parallel computing: (1) (2) (3) (4) & & & $\boldsymbol{V}$ \\
\hline & $\begin{array}{l}\text { e. Having background knowledge and understanding of computer } \\
\text { science concepts (e.g. parallel computing) is valuable in and of itself: } \\
\text { (1) (2) (3) (4) }\end{array}$ & & $\boldsymbol{V}$ & $\boldsymbol{V}$ \\
\hline & $\begin{array}{l}\text { 4. I was interested to interact with SeeMore because ( you can select } \\
\text { more than } 1 \text { option) }\end{array}$ & & & \\
\hline & a. I like to learn computer related stuff & & $\boldsymbol{V}$ & $\checkmark$ \\
\hline & b. I did not know what parallel computing is & $\checkmark$ & & $\checkmark$ \\
\hline & c. The sculpture looked cool and I just wanted to know what it was & $\boldsymbol{V}$ & & $\checkmark$ \\
\hline & d. I was with a friend and I came along & $\boldsymbol{V}$ & & \\
\hline \multirow{14}{*}{ 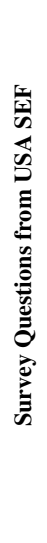 } & 5. I learned something new from SeeMore. & $\boldsymbol{v}$ & & \\
\hline & 6. I enjoyed my interaction with SeeMore. & $\boldsymbol{V}$ & & \\
\hline & 7. I would like to see future exhibits with SeeMore. & $\boldsymbol{V}$ & & \\
\hline & 8. Students would learn something new from SeeMore. & $\boldsymbol{V}$ & & \\
\hline & 9. Students would enjoy interacting with SeeMore. & $\boldsymbol{V}$ & & \\
\hline & 10. Students would be motivated to learn from SeeMore. & $\boldsymbol{V}$ & $\checkmark$ & $\checkmark$ \\
\hline & 11. SeeMore has educational value. & $\boldsymbol{V}$ & & \\
\hline & $\begin{array}{l}\text { 12. SeeMore would get students interested in STEAM (Science, } \\
\text { Technology, Engineering, Art, and Mathematics) careers. }\end{array}$ & $\checkmark$ & $\boldsymbol{v}$ & $\boldsymbol{V}$ \\
\hline & $\begin{array}{l}\text { 13. Please rank each of the following items... with \#1 being the most } \\
\text { important to \#5 being the least important. }\end{array}$ & & & \\
\hline & a. SeeMore is interactive & $\boldsymbol{V}$ & & $\checkmark$ \\
\hline & b. SeeMore is a movable sculpture & $\boldsymbol{V}$ & & $\checkmark$ \\
\hline & c. SeeMore combines art and computer science & $\boldsymbol{V}$ & $\boldsymbol{V}$ & $\checkmark$ \\
\hline & d. SeeMore is a 3D representation of a concept & $\boldsymbol{V}$ & $\checkmark$ & $\checkmark$ \\
\hline & e. SeeMore makes a complicated idea easier to understand & $\checkmark$ & $\boldsymbol{V}$ & $\checkmark$ \\
\hline
\end{tabular}


SeeMore at conferences with total attendance over 600,000 people of diverse backgrounds including attendees at:

1. The annual conference of the ACM Special Interest Group on Computer Graphics and Interactive Techniques Conference (ACM SIGGRAPH '14), Vancouver, Canada, August 10-14, 2014 (1̃5,000 attendees);

2. The annual International Maker Faire (IMF), New York Hall of Science, New York City, New York, September 21-27, 2015 (9̃0,000 attendees);

3. The annual South By Southwest (SXSW 2016) Interactive Conference, Austin, Texas, March 11-20, 2016 (1̃50,000 attendees); and

4. The biennial U.S.A. Science and Engineering Festival (SEF), Washington, D.C., April 15-19, 2016 ( $\tilde{3} 65,000$ attendees).

Exhibit participants viewed a short 1-2 minute live SeeMore demonstration followed by a Q\&A session with members of the technical teams. We did not conduct formal surveys of SIGGRAPH and SXSW audiences for logistical reasons. Upon completion of the demonstration, audience members at IMF and SEF were asked to fill out a survey to gauge their experience with SeeMore. Table 1 shows the survey questions provided to participants for IMF and SEF respectively. This table also shows how the survey questions are related to evaluating the three identified project objectives.

For interactive exhibits, Creswell [6] suggests the use of interviews to assess the experience of a participant. For our qualitative analyses, we assess the responses from survey participants following both our own objectives [O1, O2, O3] and Stevenson's taxonomy [30] which includes classification of six types of learning experiences for interactive exhibits: 1) a set of experiences such as what they did, felt or thought;2) a set of effects such as noticing patterns in the sculpture; 3) a set of derived explanations for the observed effects; 4) a set of applications such as anticipating how the sculpture might benefit others; 5) an improved understanding of parallel computation; and 6) a change in attitude.

\subsubsection{Analysis of IMF Interviews and Surveys (September 2015)}

The IMF audience is well studied and according to makermedia.com, a typical audience at the large IMF conferences in New York, NY and San Jose, CA have the fol- 
lowing demographics: "They tend to be male, home owners, and married with children; their median age is 44 . Most are employed (82\%), 16\% are self-employed. Primarily male $(70 \%)$. Two-thirds $(66 \%)$ own their own home or apartment. Over two-thirds are married or living as married (69\%). Over four in ten (43\%) attended Maker Faire with displayed aided their understanding [Table 2, Q3b] while $68 \%$ enjoyed the experience [Table 2, Q3c]. These results would indicate direct, strong support for a key objective of the SeeMore project: to entertain and educate all audiences to improve the PDC pipeline [O1].

535 
Table 2: IMF 2015 Survey Results. Survey summary results for 63 participants from the annual International Maker Faire (IMF), New York Hall of Science, New York City, New York, September 21-27, 2015 ( 90,000 attendees). Participants were asked to fill out surveys after a short 1-2 minutes demonstration and Q\&A with the SeeMore design team.

[63 PARTICIPANTS SURVEYED]

Q1. Prior to interacting with SeeMore how familiar we
\begin{tabular}{|c|c|c|c|c|} 
a. Not at all familiar; b. Slightly samiliar; c. Somewhat \\
\hline $\mathbf{a}$ & $\mathbf{b}$ & $\mathbf{c}$ & $\mathbf{d}$ & $\mathbf{e}$ \\
$\mathbf{2 5}$ & 15 & 6 & 13 & 4 \\
$\mathbf{3 9 . 7 \%}$ & $\mathbf{2 3 . 8 \%}$ & $9.5 \%$ & $20.6 \%$ & $6.3 \%$ \\
\hline
\end{tabular}

Q2. Now that you have interacted with SeeMore, how would you rate your level of understanding of parallel computing: a. Poor; b. Fair; c. Good; d. Very good; e. Excellent

\begin{tabular}{c|c|c|c|c|}
$\mathbf{a}$ & $\mathbf{b}$ & $\mathbf{c}$ & $\mathbf{d}$ & $\mathbf{e}$ \\
4 & 15 & 26 & 10 & 8 \\
$6.3 \%$ & $23.8 \%$ & $\underline{\mathbf{4 1 . 3 \%}}$ & $\underline{\mathbf{1 5 . 9 \%}}$ & $\underline{\mathbf{1 2 . 7 \%}}$ \\
\hline
\end{tabular}

Q3. Indicate whether you (1) strongly agree, (2) agree, (3) disagree, or (4) strongly disagree with the following statements (Circle/Cross One):

a. I now better understand how things work in parallel: (1) (2) (3) (4)

\begin{tabular}{|c|c|c|c|l|}
\hline $\mathbf{1}$ & $\mathbf{2}$ & $\mathbf{3}$ & $\mathbf{4}$ & \\
18 & 26 & 15 & 4 & \\
$\mathbf{2 8 . 6 \%}$ & $\mathbf{4 1 . 3 \%}$ & $23.8 \%$ & $6.3 \%$ & \\
\hline
\end{tabular}

b. The computational patterns displayed by SeeMore helped my visual understanding of parallel computing: (1) (2) (3) (4)

\begin{tabular}{|c|c|c|c|}
$\mathbf{1}$ & $\mathbf{2}$ & $\mathbf{3}$ & $\mathbf{4}$ \\
$\mathbf{2 4}$ & 16 & 13 & 10 \\
$\mathbf{3 8 . 1} \%$ & $\mathbf{2 5 . 4 \%}$ & $20.6 \%$ & $15.9 \%$ \\
\hline
\end{tabular}

c. I enjoyed interacting with SeeMore: (1) (2) (3) (4)

\begin{tabular}{c|c|c|c|c|}
$\mathbf{1}$ & $\mathbf{2}$ & $\mathbf{3}$ & $\mathbf{4}$ & \\
$\mathbf{5 4 . 0 \%}$ & 9 & 4 & 16 \\
$\mathbf{1 4 . 3 \%}$ & $6.3 \%$ & $25.4 \%$ \\
\hline
\end{tabular}

d. I would like to learn more about parallel computing: (1) (2) (3) (4)

\begin{tabular}{|c|c|c|c|l|}
\hline $\mathbf{1}$ & $\mathbf{2}$ & $\mathbf{3}$ & $\mathbf{4}$ & \\
$\mathbf{3 2}$ & 9 & 10 & 12 & \\
$\mathbf{5 0 . 8 \%}$ & $\mathbf{1 4 . 3 \%}$ & $15.9 \%$ & $19.0 \%$ & \\
\hline
\end{tabular}

e. Having background knowledge and understanding of computer science concepts (e.g. parallel computing) is valuable in and of itself: (1) (2) (3) (4)

\begin{tabular}{c|c|c|c|c|}
$\mathbf{1}$ & $\mathbf{2}$ & $\mathbf{3}$ & $\mathbf{4}$ & \\
32 & 14 & 6 & 11 & \\
$\mathbf{5 0 . 8 \%}$ & $\mathbf{2 2 . 2 \%}$ & $9.5 \%$ & $17.5 \%$ & \\
\hline
\end{tabular}

Q4. I was interested to interact with SeeMore because (you can select more than 1 option)

a. I like to learn computer related stuff; b. I did not know what parallel computing is; $c$. The sculpture looked cool and I just wanted to know what it was; d. I was with a friend and I came along

\begin{tabular}{c|c|c|c|c}
$\mathbf{a}$ & $\mathbf{b}$ & $\mathbf{c}$ & $\mathbf{d}$ & \\
$\underline{\mathbf{4 4}}$ & 24 & $\underline{\mathbf{4 2}}$ & 15 & \\
$\mathbf{6 9 . 8} \%$ & $38.1 \%$ & $\mathbf{6 6 . 7} \%$ & $23.8 \%$ & [\% participants that selected this choice] \\
\hline
\end{tabular}

Q5. Are you currently:

a. A student; b. Employed for wages; c. Self-employed; d. Not working; e. A homemaker; f. Military; g. Retired; h. Other

\begin{tabular}{c|c|c|c|c|c|c}
$\mathbf{a}$ & $\mathbf{a , b}$ & $\mathbf{b}$ & $\mathbf{c}$ & $\mathbf{d}$ & $\mathbf{g}$ & [only combinations selected by participants shown] \\
35 & 1 & 20 & 4 & 1 & 2 & \\
$\mathbf{5 5 . 6 \%}$ & $1.6 \%$ & $31.7 \%$ & $6.3 \%$ & $1.6 \%$ & $3.2 \%$ & [\% participants that selected this choice or combination]
\end{tabular}

Q6. Are you currently:

a. Under 10 years of age; b. Between 11 and 17 years of age; c. Between 18 and 30 years of age; d. Between 31 and 40 years of age; e. Between 41 and 50 years of age; f. Between 50 and 65 years of age; g. Over 65 years of age

\begin{tabular}{|c|c|c|c|c|c|}
\hline $\mathbf{a}$ & $\mathbf{b}$ & $\mathbf{c}$ & $\mathbf{d}$ & $\mathbf{e}$ & \\
4 & 20 & 23 & 5 & 9 & \\
$\mathbf{6 . 6 \%}$ & $\mathbf{3 2 . 8 \%}$ & $\underline{\mathbf{3 7 . 7 \%}}$ & $8.2 \%$ & $14.8 \%$ & \\
\hline
\end{tabular}


interaction with the kinetic sculpture would reinforce a belief that parallel computation provides value and impact on an individual's life. A supermajority (more than 73\%) "agreed" or "strongly agreed" that having background knowledge and understanding of computer science concepts is valuable [Table 2, Q3e]. This would appear to support our objective [O2] to "personalize the importance of parallel computation."

In a related question, as evidence of the potential for SeeMore to improve the PDC pipeline, participants were asked whether they would like to learn more about parallel computing. Survey results indicate that more than $65 \%$ were inspired to learn more [Table 2, Q3d]. This supports our project objective [O3] to "inspire participants to learn more."

At IMF, we also conducted video interviews with 11 attendees after they interacted with SeeMore. Each interview ran from 1-3 minutes and participants were asked to share their impressions or what they learned from SeeMore. Table 3 provides select quotes from these interviews. We interviewed 7 males and 4 females varying in age from about 6 to 40 including 2 that identified as Hispanic and 1 that identified as African American. These participants were universally positive and their comments skewed heavily in support of the entertainment and educational value of SeeMore to improve the PDC pipeline [O1] with quotes such as "great for teaching parallel computing" and "cool because it's a bunch of computers working together." Participants were inspired to learn more [O3] with comments from "it's beautiful" and "really inspiring" to "[I was] inspired to do more research on a topic I had given up on." Participants also personalized the experience [O2] commenting the exhibit was particularly "useful because you related it to things like Facebook and Google" and that they "hope you guys do more work" like SeeMore.

Using Stevenson's taxonomy [30] these interviews show that the interactive nature of the SeeMore exhibit led to memorable experiences (e.g., "a fantastic sculpture representation that the kids would love") for most participants interviewed. These experiences led to a number of different effects such as new ways of thinking about parallel computation (e.g., "there is this wonderful connection between the computers and the work they're doing") and perceived applications of these effects (e.g., "great for teaching parallel computing"). Attitudinal changes were also common in participants from 
Table 3: IMF 2015 Video Interview Results. Select quotes from video interviews during the IMF exhibition. Participants were asked to provide additional comments about what they learned or would like to share about their interaction with SeeMore. Quotes are evaluated in relation to both the three project objectives and the six types of experience classifications of the Stevenson taxonomy. $[O 1=$ Entertain and educate all audiences, $\mathrm{O} 2=$ Personalize the importance of parallel computation, $\mathrm{O} 3=$ Inspire participants to learn more.]

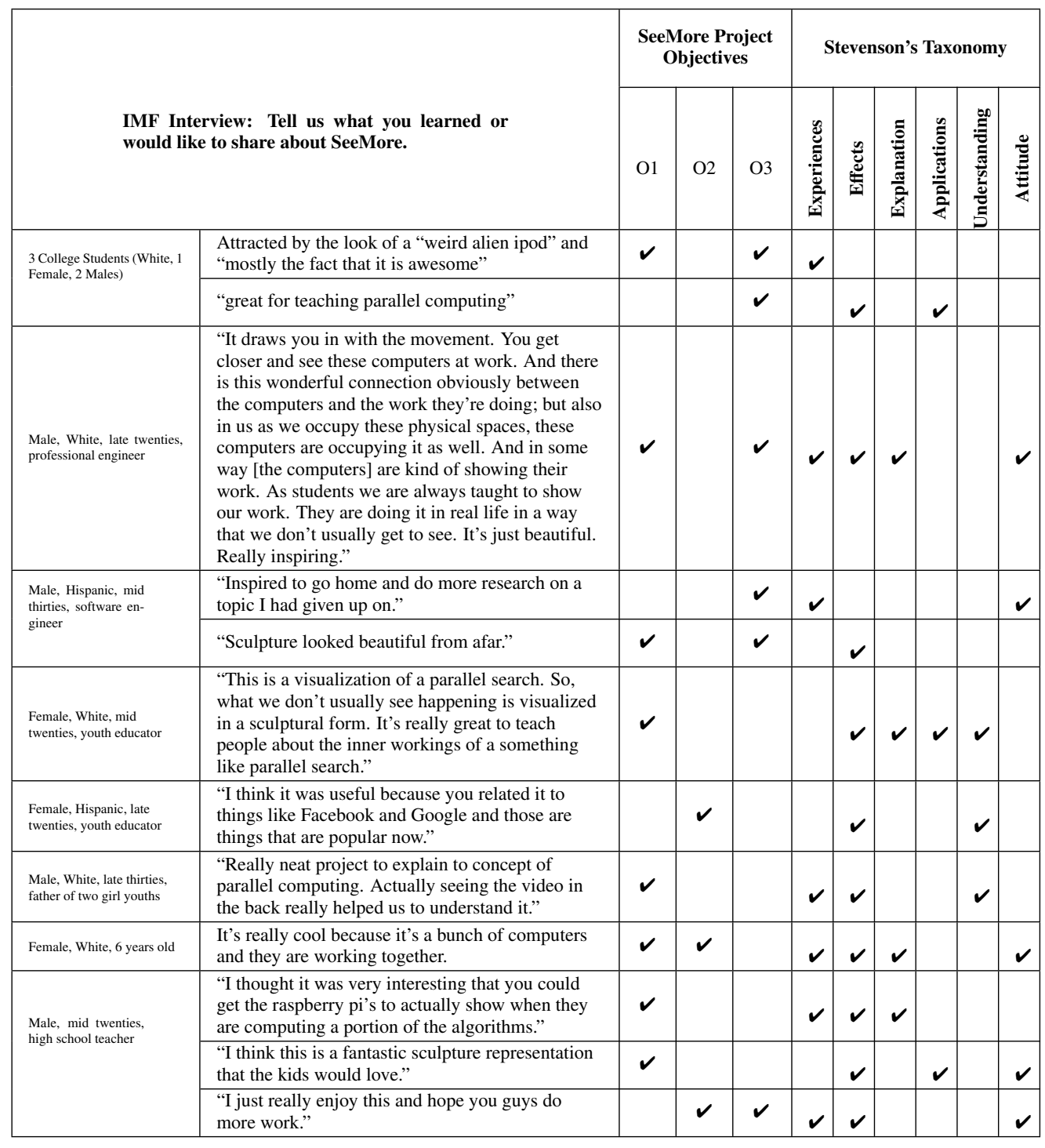


pure enjoyment (e.g., "I just really enjoy this") to inspiration (e.g., "really inspiring”) and cool factor (e.g., "really neat", "really cool", "awesome").

\subsubsection{Analysis of SEF Surveys (April 2016)}

For the SEF conference, we implemented some changes with respect to the IMF exhibit. First, we determined to increase survey participation in anticipation of the larger expected crowds. Second, we added a microphone and speaker system to handle larger crowds for each demonstration. Third, we modified the questions in the survey in anticipation of a higher concentration of students and teachers typical at a science and engineering faire so as to gauge the potential affects on the PDC pipeline. We also added an open-ended comment question to the survey to replace video interviews logistically impracticable due to the high throughput of visitors expected.

The biennial USA Science and Engineering Festival (SEF) attracts 365,000 K-12 students, teachers, families, education professionals, government officials and press to the Washington D.C. convention center located a few city blocks from the White House. According to usasciencefestival.org, the SEF serves as an open forum to showcase all facets of Science, Technology, Engineering, and Math. Attendees hail from all 50 states including over 2,000 participating schools and more than 5,000 students and teachers from underserved schools. SeeMore was a featured exhibit invited and sponsored by the National Science Foundation (NSF) for inclusion as part of the NSF exhibition.

We estimate 12,000-15,000 or more SEF attendees visited the SeeMore exhibit during the 3-day event. Of these, 100 individuals participated in the SEF survey. Table 4 provides a summary of SEF survey results. The participating audience is as advertised and skews younger than the IMF audience with more than 50\% reporting as 17 and under [Table 4, Q2] and 50\% identifying themselves as K-12 students [Table 4. Q4]. Another $30 \%$ was about evenly distributed from 31 to 65 years of age with a nearly matching 33\% overall identifying themselves as parents or other (often retirees). This data supports the assumption that the majority of attendees were groups of students and teachers or extended families with children and often including grandparents. The audience was fairly diverse in race with $40.6 \%$ identifying as white, $32.3 \%$ identifying as Asian, and $14.6 \%$ identifying as African American [Table 4, Q3]. Overall, the 
Table 4: SEF 2016 Survey Results. Survey summary results for 100 participants from the biennial U.S.A. Science and Engineering Festival (SEF), Washington, D.C., April 15-19, 2016 (365,000 attendees). Participants were asked to fill out surveys after a short 1-2 minutes demonstration and Q\&A with the SeeMore design team.

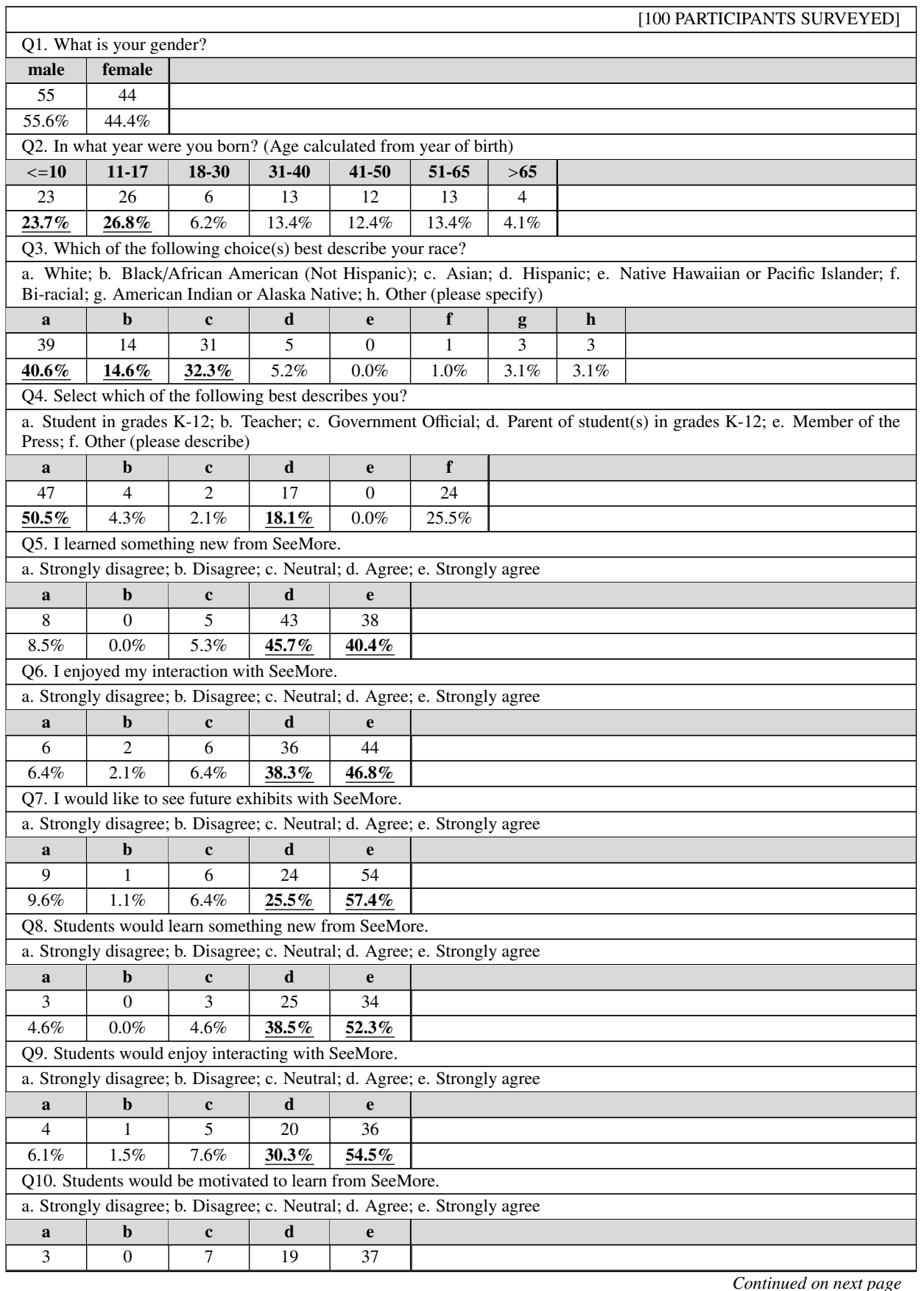


Table 4 - Continued from previous page

\begin{tabular}{|c|c|c|c|c|c|c|}
\hline $4.5 \%$ & $0.0 \%$ & $10.6 \%$ & $\underline{28.8 \%}$ & $\underline{56.1 \%}$ & & \\
\hline \multicolumn{7}{|c|}{ Q11. SeeMore has educational value. } \\
\hline \multicolumn{7}{|c|}{ a. Strongly disagree; b. Disagree; c. Neutral; d. Agree; e. Strongly agree } \\
\hline a & b & c & d & e & & \\
\hline 3 & 0 & 5 & 24 & 34 & & \\
\hline $4.5 \%$ & $0.0 \%$ & $7.6 \%$ & $\underline{36.4 \%}$ & 51.5\% & & \\
\hline \multicolumn{7}{|c|}{ Q12. SeeMore would get students interested in STEAM (Science, Technology, Engineering, Art, and Mathematics) careers. } \\
\hline \multicolumn{7}{|c|}{ a. Strongly disagree; b. Disagree; c. Neutral; d. Agree; e. Strongly agree } \\
\hline a & b & c & d & $\mathbf{e}$ & & \\
\hline 6 & 0 & 4 & 24 & 31 & & \\
\hline $9.2 \%$ & $0.0 \%$ & $6.2 \%$ & $\underline{36.9 \%}$ & $\underline{47.7 \%}$ & & \\
\hline \multicolumn{7}{|c|}{ Q13. Please rank each of the following items... with \#1 being the most important to \#5 being the least important. } \\
\hline \multicolumn{7}{|c|}{$\begin{array}{l}\text { a. SeeMore is interactive; b. SeeMore is movable sculpture; c. SeeMore combines art and computer science; d. SeeMore is } \\
\text { a 3D representation of a concept; e. SeeMore makes a complicated idea easier to understand. }\end{array}$} \\
\hline & a & b & c & d & & $\begin{array}{l}\text { [Note: Some participants expressed equal importance } \\
\text { to items by using the same ranking for mulitple items.] }\end{array}$ \\
\hline 1 & 8 & 12 & 16 & 12 & 18 & Votes for $(a-b-c-d-e)$ most important. \\
\hline 2 & 8 & 6 & 4 & 15 & 12 & \\
\hline 3 & 11 & 17 & 30 & 39 & 45 & \\
\hline 4 & 12 & 18 & 26 & 33 & 40 & \\
\hline 5 & 12 & 32 & 41 & 48 & 55 & Votes for $(a-b-c-d-e)$ least important \\
\hline
\end{tabular}

surveyed audience was $55.6 \%$ male and $44.4 \%$ female [Table 4 , Q1].

Following their interaction with SeeMore, more than $86 \%$ "agreed" or "strongly agreed" that they had learned something new from SeeMore [Table 4, Q5]. More than 90\% of respondents "agreed" or "strongly agreed" that students would learn something new from SeeMore [Table 4, Q8]. An overwhelming majority (87.9\%) "agreed" or "strongly agreed" that SeeMore has educational value [Table 4, Q11]. This data directly affirms part of our objective [O1] of educating all audiences to improve the PDC pipeline.

More than $85 \%$ of survey participants, "agreed" or "strongly agreed" that they had enjoyed their interaction with SeeMore [Table 4, Q6]. More than $84 \%$ of respondents "agreed" or "strongly agreed" that students would enjoy interacting with SeeMore [Table 4. Q9]. This data directly affirms part of our objective [O1] of entertaining all audiences to improve the PDC pipeline. This data also indirectly supports our objective [O2] of getting attendees to personalize the importance of parallel computation. For example, the belief that SeeMore has educational value likely results from internalizing the perceived value of interaction with the sculpture.

We asked survey participants to rank their perceived relative importance of items 
(i.e. characteristics) related to their interactions and impressions from the SeeMore exhibit [Table 4, Q13]. Respondents were to rank SeeMore's: a) interactivity, b) movability, c) combined art and science aspect, d) 3D representation of a concept; and e) ability to convey a complex idea simply. The results here gave no clear indication of the overall relative importance of these characteristics. This question seems to ascertain individual's beliefs about the relative importance of aspects of SeeMore related to parallel computation [O2]. The results would seem to confirm participants found different aspects more important than others without a clear consensus across the broad audience.

Following their interaction with SeeMore, $85.1 \%$ of respondents "agreed" or "strongly agreed" they would like to see future exhibits with SeeMore [Table 4, Q7]. More than $84 \%$ of individuals surveyed felt that SeeMore would motivate others to learn as well [Table 4, Q10]. Perhaps most importantly, 84.6\% of respondents believe SeeMore would get students interested in careers that integrate Science, Technology, Engineering, Art, and Mathematics (STEAM fields) [Table 4, Q12] - more pointedly, this would indicate SeeMore exposure would inspire students to enter the PDC pipeline. These results seem to indicate that participants believe they and others would be inspired to learn more from SeeMore and about parallel computation [O3].

As mentioned, we also asked participants to share any additional comments in survey Question 14 (see Table 5). There were 16 respondents ( 8 male and 8 female) to this question in total from the ages of 9 to 65 . Of these participants, 5 identified as white, 8 identified as Asian, 2 identified as African American, and 1 identified as American Indian or Alaska Native.

As in the IMF interview results, these comments were overwhelmingly positive. The majority provides supporting evidence for meeting the project goal of entertaining and educating all audiences to improve the PDC pipeline [O1]. Examples include comments on the clarity of the educational demo (e.g., "very visual and easy to understand") and comments regarding what was learned (e.g., "demo/talk about datacenter architecture").

Respondents also made comments relevant to personalizing the importance and relevance of parallel computation [O2]. Responses varied from those advocating for 
Table 5: SEF 2016 Q14 Survey Results. Select quotes from Question \#14 in the SEF survey. Participants were asked to provide additional comments or questions related to their interaction with SeeMore. Quotes are evaluated in relation to both the three project objectives and the six types of experience classifications of the Stevenson taxonomy. $[O 1=$ Entertain and educate all audiences, $O 2=$ Personalize the importance of parallel computation, $\mathrm{O} 3=$ Inspire participants to learn more.]

\begin{tabular}{|c|c|c|c|c|c|c|c|c|c|c|}
\hline \multirow{2}{*}{\multicolumn{2}{|c|}{$\begin{array}{l}\text { USA SEF Question 14: Share any addi- } \\
\text { tional comments or questions you have about } \\
\text { SeeMore. }\end{array}$}} & \multicolumn{3}{|c|}{$\begin{array}{l}\text { SeeMore Project } \\
\text { Objectives }\end{array}$} & \multicolumn{6}{|c|}{ Stevenson's Taxonomy } \\
\hline & & \multirow{2}{*}{$\frac{\mathrm{O} 1}{\boldsymbol{v}}$} & \multirow{2}{*}{$\mathrm{O} 2$} & \multirow{2}{*}{$\mathrm{O} 3$} & \multirow{2}{*}{ 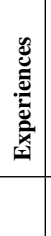 } & \multirow{2}{*}{ 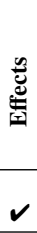 } & \multirow{2}{*}{ 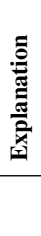 } & \multirow{2}{*}{$\frac{\mathscr{a}}{\stackrel{0}{0}}$} & \multirow{2}{*}{ 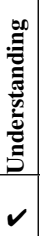 } & \multirow{2}{*}{ 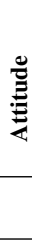 } \\
\hline Male, White, 39 & $\begin{array}{l}\text { "Demo/talk about datacenter } \\
\text { architecture" }\end{array}$ & & & & & & & & & \\
\hline Male, White, 11 & “Awesome!!!” & $\checkmark$ & & & & $\boldsymbol{V}$ & & & & $\boldsymbol{V}$ \\
\hline Male, Asian, 12 & $\begin{array}{l}\text { "How much processing power does } \\
\text { SeeMore have?" }\end{array}$ & & & $\checkmark$ & & $\checkmark$ & & & & \\
\hline Female, Asian, 12 & "It was fun." & $\boldsymbol{v}$ & & & $\boldsymbol{\sim}$ & $\boldsymbol{V}$ & & & & $\boldsymbol{V}$ \\
\hline Female, White, 13 & "It was very interesting." & $\checkmark$ & & & & $\boldsymbol{v}$ & & & & $\boldsymbol{V}$ \\
\hline Female, Asian, 9 & "SeeMore is very cool and amazing." & $\boldsymbol{v}$ & & & & $\checkmark$ & & & & $\boldsymbol{V}$ \\
\hline $\begin{array}{l}\text { Male, American Indian or } \\
\text { Alaska Native, } 65\end{array}$ & $\begin{array}{l}\text { "I am curious as to its cost, and how } \\
\text { Moore's law affects its cost ever time" }\end{array}$ & & & $\checkmark$ & & $\boldsymbol{V}$ & & $\boldsymbol{V}$ & & \\
\hline Male, White, 11 & "Interesting" & $\checkmark$ & & & & $\boldsymbol{V}$ & & & & $\boldsymbol{V}$ \\
\hline $\begin{array}{l}\text { Female, Black/African } \\
\text { American, } 9\end{array}$ & "Why is SeeMore moving like that?" & & & $\boldsymbol{v}$ & & $\boldsymbol{V}$ & & & & \\
\hline $\begin{array}{l}\text { Female, Black/African } \\
\text { American, } 12\end{array}$ & "Why do they move in and out?" & & & $\checkmark$ & & $\boldsymbol{V}$ & & & & \\
\hline Female, Asian, 11 & $\begin{array}{l}\text { "It's interesting that SeeMore is } 250 \mathrm{x} \text { as } \\
\text { cool as a computer!" }\end{array}$ & $\boldsymbol{v}$ & & & & $\checkmark$ & & & $\boldsymbol{\nu}$ & \\
\hline Female, White, 47 & "Please fund SeeMore!" & & $\checkmark$ & & & & & & & \\
\hline Male, Asian, 38 & $\begin{array}{l}\text { "Love the art, love the concept. Would } \\
\text { help to show more computer use cases } \\
\text { through some art exhibit" }\end{array}$ & $\boldsymbol{v}$ & & $\checkmark$ & & $\boldsymbol{V}$ & & $\boldsymbol{v}$ & & $\boldsymbol{V}$ \\
\hline Male, Asian, 34 & "Fellow hokie, great job!" & & & & & $\boldsymbol{v}$ & & & & \\
\hline Female, Asian, 21 & $\begin{array}{l}\text { "Thank you for bringing and building } \\
\text { SeeMore. Would be cool if someone } \\
\text { allows users to utilize the results on the } \\
\text { actual SeeMore boards." }\end{array}$ & & & $\checkmark$ & & $\boldsymbol{V}$ & & $\boldsymbol{v}$ & & \\
\hline Male, Asian, 10 & $\begin{array}{l}\text { "Very visual and easy to understand. } \\
\text { Very userful to kids and relevant" }\end{array}$ & $\boldsymbol{v}$ & $\boldsymbol{v}$ & & & $\checkmark$ & & $\boldsymbol{v}$ & $\boldsymbol{v}$ & \\
\hline
\end{tabular}


more funding for projects like SeeMore (e.g., "please fund SeeMore") to comments related to the usefulness of SeeMore-like education for children (e.g., "very useful to kids and relevant").

SeeMore also inspired participants at SEF to learn more about parallel computation [O3]. These responses often came in the form of questions such as: "How much processing power does SeeMore have?" and "How [does] Moore's law affect its cost over time?" Others wrote questions that are frequently asked of technical team members at SeeMore exhibitions. Examples include: questions such as "Why is SeeMore moving like that?" and suggestions such as "Would [like to see] more computer use cases".

Using Stevenson's taxonomy [30] these comments suggest that the interactive nature of the SeeMore exhibit led to memorable experiences (e.g., "Awesome!", "It was fun.", "It was very interesting.") for several respondents. These experiences led to a number of different effects such as deeper questions about the design and use of SeeMore (e.g., "Would be cool if someone allows users to utilize the results on the actual SeeMore boards.") and perceived applications of these effects (e.g., "very useful to kids"). Attitudinal changes were also common in participants from pure enjoyment (e.g., "It was fun.") to inspiration (e.g., "SeeMore is $250 x$ as cool as a computer") and cool factor (e.g., "awesome" "cool", "great job").

\section{Conclusions and Future Work}

We presented the design, implementation and evaluation of the SeeMore kinetic parallel cluster. Our primary objectives were to entertain and educate broad audiences to improve the PDC pipeline, convince people of the importance of parallel computing to their everyday lives, and inspire participants to learn more. At both the IMF and SEF conferences, all of these objectives were met overwhelmingly based upon survey data from over 150 participants from approximately 15,000-20,000 people that experienced these SeeMore exhibitions.

While previous work indicates that the use of kinetic art and visualizations for teaching has limits, our work demonstrates that kinetic visualizations of parallel algorithms can impart a basic understanding of the beauty, complexity, and importance 
of parallel computation to very broad audiences. Limitations of these studies include the size of the sample survey data set relative to the number of attendees and the demographics of the sample pool that consists primarily of individuals with at least a passing interest in science and technology. These limitations are somewhat alleviated by the combination of quantitative analyses via surveys and qualitative analyses via interviews and videos. Furthermore, the survey results for many questions were overwhelmingly favorable for key findings such as the percentage of survey respondents that enjoyed their interaction with SeeMore (63\% at IMF, $84 \%$ at SEF) and the percentage that believe SeeMore has educational value (69\% at IMF, $87 \%$ at SEF). The almost $20 \%$ difference between respondents at IMF and SEF for these questions may indicate SeeMore was found particularly entertaining and valuable to the younger SEF audience or that the revised SEF demo was more effective than the previous version. Moreover, this would indicate SeeMore is an excellent tool to improve youth interest in parallel and distributed computing (PDC).

The SeeMore project has been a fantastic experiment to excite people of all ages and backgrounds about parallel computing. From this perspective, SeeMore has been a tremendous success and we hope this success will encourage others to consider creative ways of exciting the public about computer science topics. In the future, we would like to integrate SeeMore with modules being taught in schools so that teachers could prepare the students for their interactions to potentially increase absorption of the key concepts. We would also like to explore other ways of creating computer artifacts that combine parallel computing technologies with art for the purpose of education. Until everyone understands the importance of parallel computing to our every day lives our work will not be complete.

\section{Acknowledgments}

The authors would like to thank Sergio Bernales, Kelsey Fahrenholtz, Timmy Meyer, Ali Butt, Bushra Tawfiq Chowdhury, and Robert Redfearn for their participation in this project. This material is based upon work supported in part by the National Science Foundation under Grant No. 1355955. 
[1] Blanchard, S., Cameron, K., 2014. Seemore, in: ACM SIGGRAPH 2014 Art Gallery, ACM, New York, NY, USA. pp. 414-415. URL: http://doi .acm. org/10.1145/2601080.2601091, doi:10.1145/2601080.2601091

[2] Brunvand, E., 2013. Arts/tech collaboration with embedded systems and kinetic art, in: ACM SIGGRAPH 2013 Talks, ACM, New York, NY, USA. pp. 23:1-23:1. URL: http://doi.acm.org/10.1145/2504459.2504488, doi: $10.1145 / 2504459.2504488$

[3] Brunvand, E., Stout, P., 2011. Kinetic art and embedded systems: A natural collaboration, in: Proceedings of the 42Nd ACM Technical Symposium on Computer Science Education, ACM, New York, NY, USA. pp.

口 323-328. URL: http://doi.acm.org/10.1145/1953163.1953263, doi:10. $1145 / 1953163.1953263$

[4] Chowdhury, B.T., Blanchard, S., Cameron, K.W., Johri, A., 2015. Seemore: An interactive kinetic sculpture designed to teach parallel computational thinking, in: 2015 ASEE Annual Conference and Exposition, ASEE Conferences, Seattle, Washington. Https://peer.asee.org/24697.

[5] Chung, C.J.C.J., 2014. Integrated steam education through global robotics art festival (graf), in: Integrated STEM Education Conference (ISEC), 2014 IEEE, pp. 1-6. doi 10.1109/ISECon.2014.6891011

[6] Creswell, J.W., 2013. Research design: Qualitative, quantitative, and mixed methods approaches. Sage publications.

[7] Dean, J., Ghemawat, S., 2008. Mapreduce: simplified data processing on large clusters. Communications of the ACM 51, 107-113.

[8] Diehl, S., 2014. Past, present, and future in and of software visualization, in: Computer Graphics Theory and Applications (GRAPP), 2014 International Conference on, pp. 1-1. 
[9] Egan, M.H., McDonald, C., 2014. Program visualization and explanation for novice C programmers, in: Proceedings of the Sixteenth Australasian Computing Education Conference - Volume 148, Australian Computer Society, Inc., Darlinghurst, Australia, Australia. pp. 51-57. URL: http://dl.acm.org/ citation. . fm?id=2667490.2667496

[10] Finlayson, I., Mueller, J., Rajapakse, S., Easterling, D., 2015. Introducing tetra: An educational parallel programming system, in: Parallel and Distributed Processing Symposium Workshop (IPDPSW), 2015 IEEE International, pp. 746751. doi:10.1109/IPDPSW.2015.51.

[11] Fouh, E., Karavirta, V., Breakiron, D.A., Hamouda, S., Hall, S., Naps, T.L., Shaffer, C.A., 2014. Design and architecture of an interactive etextbook the opendsa system. Science of Computer Programming 88, 22

- 40. URL: http://wwW.sciencedirect.com/science/article/pii/ S016764231300333X, doi $10.1016 /$ j.scico.2013.11.040. software Development Concerns in the e-Learning Domain.

[12] Gong, C., Liu, J., Gong, Z., Qin, J., Xie, J., 2010. Algorithms and Architectures for Parallel Processing: 10th International Conference, ICA3PP 2010, Busan, Korea, May 21-23, 2010. Proceedings. Part I. Springer Berlin Heidelberg, Berlin, Heidelberg. chapter Optimizing Sweep3D for Graphic Processor Unit. pp. 416-426. URL: http://dx.doi.org/10.1007/978-3-642-13119-6_36, doi:10.1007/978-3-642-13119-6_36.

[13] Guo, P.J., 2013. Online python tutor: Embeddable web-based program visualization for cs education, in: Proceeding of the 44th ACM Technical Symposium on Computer Science Education, ACM, New York, NY, USA. pp.

a 579-584. URL: http://doi.acm.org/10.1145/2445196.2445368, doi:10. $1145 / 2445196.2445368$

[14] Hahn, S., Trapp, M., Wuttke, N., Dllner, J., 2015. Thread city: Combined visualization of structure and activity for the exploration of multi-threaded software 
systems, in: 2015 19th International Conference on Information Visualisation, pp. 101-106. doi:10.1109/iV.2015.28.

[15] Hoisie, A., Lubeck, O., Wasserman, H., 1999. Workshop on wide area networks and high performance computing. Springer London, London. chapter Performance analysis of wavefront algorithms on very-large scale distributed systems. pp. 171-187. URL: http://dx.doi.org/10.1007/BFb0110087, doi: $10.1007 / \mathrm{BFb} 0110087$.

[16] HUNDHAUSEN, C.D., DOUGLAS, S.A., STASKO, J.T., 2002. A meta-study of algorithm visualization effectiveness. Journal of Visual Languages \& Computing 13, 259-290.

[17] JáJá, J., 1992. An Introduction to Parallel Algorithms. Addison Wesley Longman Publishing Co., Inc., Redwood City, CA, USA.

[18] Jee, K., Hong, E., 2015. The development and application of steam education program based on kinetic art. Journal of Korean Elementary Science Education 34, 276-287.

[19] Kang, M.s., Kang, M.H., Ha, O.K., Jun, Y.K., 2016. Conpathview: A visualization tool for debugging race conditions in event synchronization of arinc 653 applications. International Journal of Software Engineering and Its Applications $10,65-76$.

[20] Karavirta, V., Shaffer, C.A., 2013. Jsav: The javascript algorithm visualization library, in: Proceedings of the 18th ACM Conference on Innovation and Technology in Computer Science Education, ACM, New York, NY, USA. pp. 159-164. URL: http://doi .acm.org/10.1145/2462476.2462487, doi:10. $1145 / 2462476.2462487$

[21] Kechkar, A., Nair, D., Heilemann, M., Choquet, D., Sibarita, J.B., 2013. Real-time analysis and visualization for single-molecule based super-resolution

口 microscopy. PLoS ONE 8, 1-10. URL: http://dx.doi.org/10.1371\% 2Fjournal.pone.0062918, doi:10.1371/journal.pone.0062918 
[22] Kim, S.H., Phang, D., An, T., Yi, J.S., Kenney, R., Uhan, N.A., 2014. Poetic: Interactive solutions to alleviate the reversal error in studentprofessor type problems. International Journal of Human-Computer Studies 72,

a 12 - 22. URL: http://wWW.sciencedirect.com/science/article/pii/ S1071581913001341, doi $10.1016 / j . i j h c s .2013 .09 .010$

[23] Lum, E.B., Stompel, A., Ma, K.L., 2003. Using motion to illustrate static 3d shape- kinetic visualization. IEEE Transactions on Visualization and Computer Graphics 9, 115-126. URL: http://dx.doi.org/10.1109/TVCG.2003. 1196000 doi $10.1109 /$ TVCG.2003.1196000

[24] Malina, F.J., Malina, F.J., 1974. Kinetic Art: Theory and Practice: Selections from the Journal Leonardo. Dover.

[25] Mathis, M.M., Amato, N.M., Adams, M.L., 2000. A general performance model for parallel sweeps on orthogonal grids for particle transport calculations, in: Proceedings of the 14th International Conference on Supercomputing, ACM, New

a York, NY, USA. pp. 255-263. URL: http://doi . acm. org/10.1145/335231. 335256 doi $10.1145 / 335231.335256$

[26] Mustafa, B., 2013. Yass: a system simulator for operating system and computer architecture teaching and learning. European Journal of Science and Mathematics Education 1, 34-42.

[27] Neumann, R.S., Kumar, S., Shalchian-Tabrizi, K., 2013. Blast output visualization in the new sequencing era. Briefings in Bioinformat-

a ics URL: http://bib.oxfordjournals.org/content/early/2013/04/ 17/bib.bbt009.abstract, doi:10.1093/bib/bbt009

[28] Offenwanger, A., Lucet, Y., 2014. Conee: An exhaustive testing tool to support learning concurrent programming synchronization challenges, in: Proceedings of the Western Canadian Conference on Computing Education, ACM, New York, [ NY, USA. pp. 11:1-11:6. URL: http://doi.acm.org/10.1145/2597959. 2597972 doi $10.1145 / 2597959.2597972$ 
[29] Schönborn, K.J., Höst, G.E., Palmerius, K.E.L., 2016. Nano ed-

n ucation with interactive visualization. Nano Today URL: http: //wWw.sciencedirect.com/science/article/pii/S174801321600013X, doi $10.1016 / \mathrm{j}$. nantod.2015.10.006

820 [30] Stevenson, J., 1991. The longterm impact of interactive exhibits. International 口 Journal of Science Education 13, 521-531. URL: http://dx.doi.org/10. 1080/0950069910130503, doi:10.1080/0950069910130503.

[31] Thorvaldsdóttir, H., Robinson, J.T., Mesirov, J.P., 2013. Integrative genomics viewer (igv): high-performance genomics data visualization and exploration. Briefings in Bioinformatics 14, 178-192. URL: http://bib.oxfordjournals.org/content/14/2/178. abstract, doi:10.1093/bib/bbs017.

[32] Turchenko, V., Demchuk, V., 2014. Efficiency analysis of parallel routine using processor time visualization. International Journal of Computing 4, 12-18. URL: http://computingonline.net/index.php/computing/ article/view/319.

[33] Valiant, L.G., 1990. A bridging model for parallel computation. Commun. ACM 口 33, 103-111. URL: http://doi.acm.org/10.1145/79173.79181, doi 10 . $1145 / 79173.79181$

[34] Velazquez-Iturbide, J.A., Debdi, O., Esteban-Sanchez, N., Pizarro, C., 2013. Greedex: A visualization tool for experimentation and discovery learning of greedy algorithms. IEEE Transactions on Learning Technologies 6, 130-143. doi:10.1109/TLT.2013.8.

[35] Wasserman, H.J., 1996. Benchmark tests on the digital equipment corporation Alpha AXP 21164-based AlphaServer 8400, including a comparison of optimized vector and superscalar processing, in: Proceedings of the 10th International Conference on Supercomputing, ACM, New York, NY, USA. pp. 333-

口 340. URL: http://doi.acm.org/10.1145/237578.237632, doi 10.1145/ 237578.237632 
[36] Wylie, B.J.N., Bhme, D., Mohr, B., Szebenyi, Z., Wolf, F., 2010. Performance analysis of sweep3d on blue gene/p with the scalasca toolset, in: Parallel Distributed Processing, Workshops and Phd Forum (IPDPSW), 2010 IEEE International Symposium on, pp. 1-8. doi:10.1109/IPDPSW. 2010.5470816.

[37] Yilmaz, B., 2014. Art engineering and kinetic art. Journal of Arts and Humanities 850 $3,16$. 
Bo Li:

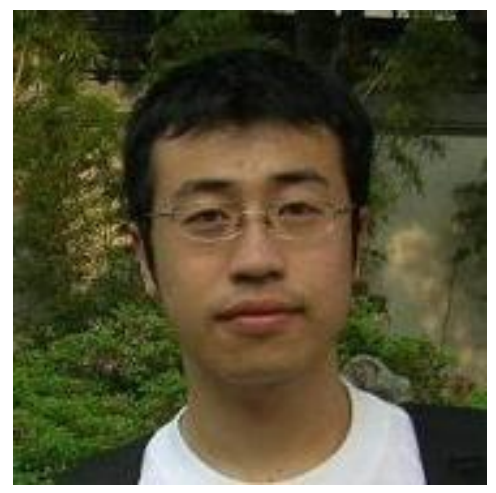

Bo Li received his BS degree in automation from Dalian University of Technology in 2006, MS degree in control theory \& control engineering from Dalian University of Technology in 2009, and MS degree in computer science from Rochester Institute of Technology in 2012. He is currently working toward the $\mathrm{PhD}$ degree at the Virginia Polytechnic Institute and State University. His research interests include power-aware computing in the high-performance computing domain and performance prediction of scientific parallel applications.

John Mooring:

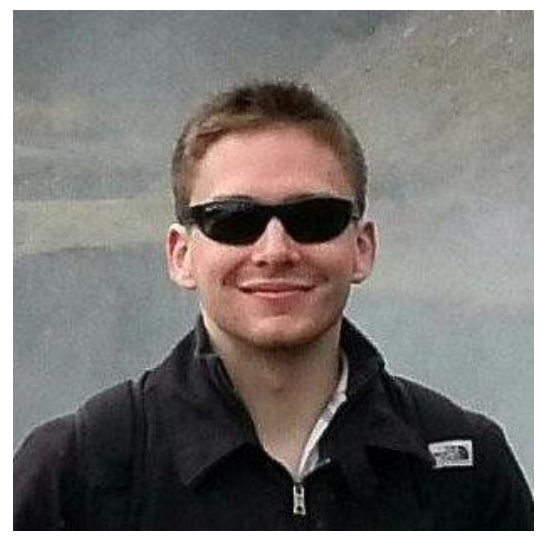

John Mooring received his BS degree in computer science from Virginia Polytechnic Institute and State University in 2014. He is currently working at a Software Engineer at Microsoft, Seattle. 
Aditya Jori:

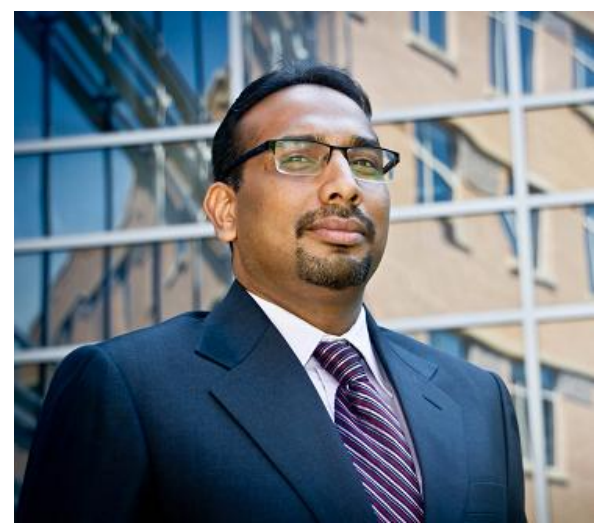

Aditya Johri, Ph.D. is an Associate Professor in the Department of Information Sciences and Technology. He studies the use of information and communication technologies (ICT) for learning and knowledge sharing, with a focus on cognition in informal environments. He also examine the role of ICT in supporting distributed work among globally dispersed workers and in furthering social development in emerging economies. He received the U.S. National Science Foundation's Early Career Award in 2009. He is co-editor of the Cambridge Handbook of Engineering Education Research (CHEER) published by Cambridge University Press, New York, NY. He earned his Ph.D. in Learning Sciences and Technology Design at Stanford University, M.S. in Information, Design, and Technology at Georgia Tech, M.A. in Mass Communication at University of Georgia, and a B.Eng. in Mechanical Engineering at Delhi College of Engineering.

Melinda Marie Leko:

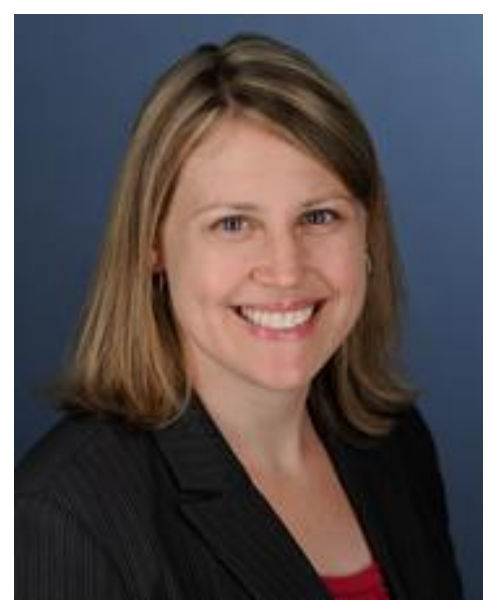

Melinda Leko is an Assistant Professor in the Department of Special Education. Her research centers on high-incidence disabilities, reading instruction, and special education teacher quality. Currently she is 
interested in better understanding how to prepare secondary teachers to deliver high quality basic reading instruction to adolescents with disabilities, particularly in inclusive classrooms. She is also interested in understanding issues of teachers' implementation of interventions. She earned her PhD at the University of Florida and worked as an Assistant Professor at the University of Wisconsin-Madison prior to becoming a faculty member at KU.Dr. Leko serves on the editorial boards for Remedial and Special Education, Journal of Teacher Education, and Intervention in School and Clinic. She served as coeditor for the special issue of Remedial and Special Education on qualitative research in special education (Volume 35 Numbers 5-6).

Sam Blanchard:

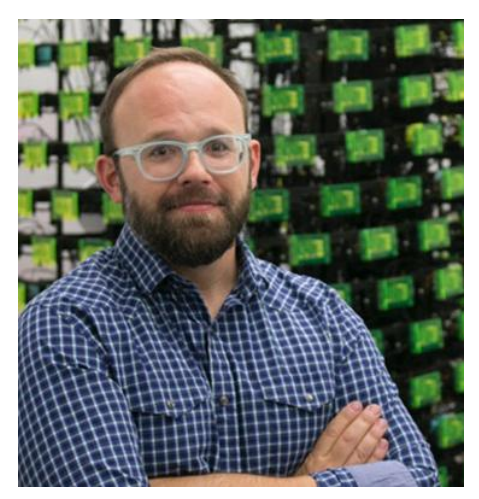

Sam Blanchard is Assistant Professor of Studio Art in the School of Visual Arts. He received his Master of Fine Art in Sculpture from the Rhode Island School of Design in 2004. Past research includes hybrid sculptures, installation, performance, and video artworks. Sam utilizes technology based fabrication methods to interweave everyday materials and objects to become extended metaphors of experience. He has an active international exhibition schedule, with solo exhibitions of his artwork at such venues as the Las Vegas Contemporary Art Center, the Delaware Center for Contemporary Art and The New Galley (Calgary, ON). Blanchard's ICAT/NSF funded collaborative projects have been featured in high profile exhibitions at the VT Center for the Arts and the SIGGRAPH Conference for Computer Graphics and Interactive Techniques. 
Kirk W. Cameron:

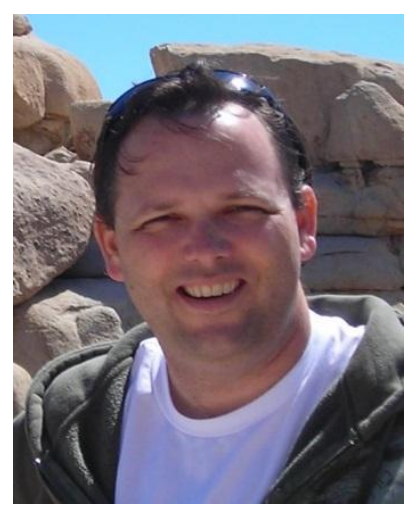

Kirk W. Cameron received the BS degree in math from UF in 1994 and the PhD degree in computer science from LSU in 2000. He is currently a professor of computer science and a Research Fellow in the College of Engineering at Virginia Tech. The central theme of his research is to improve power and performance efficiency in high performance computing (HPC) systems and applications. He is a pioneer and leading expert in Green Computing. Cameron is also the Green IT columnist for IEEE Computer, Green500 co-founder, founding member of SPECPower, EPA consultant, Uptime Institute Fellow, and co-founder of power management software startup company MiserWare. Accolades for his work include NSF and DOE Career Awards, the IBM Faculty Award, and being named Innovator of the Week by Bloomberg Businessweek Magazine. 